\title{
SCIENTIFIC REPORTS

Macrophage migration inhibitory factor of Syrian golden hamster shares structural and functional similarity with human counterpart and promotes pancreatic cancer

\author{
Voddu Suresh ${ }^{1,3,7}$, Rajivgandhi Sundaram ${ }^{2,4,7}$, Pujarini Dash ${ }^{1,7}$, Surendra Chandra Sabat ${ }^{5}$, \\ Debasish Mohapatra ${ }^{1}$, Sneha Mohanty ${ }^{6}$, Dileep Vasudevan ${ }^{2 *}$ \& Shantibhusan Senapati ${ }^{1 *}$
}

Macrophage migration inhibitory factor (MIF) is a pleiotropic cytokine that increasingly is being studied in cancers and inflammatory diseases. Though murine models have been instrumental in understanding the functional role of MIF in different pathological conditions, the information obtained from these models is biased towards a specific species. In experimental science, results obtained from multiple clinically relevant animal models always provide convincing data that might recapitulate in humans. Syrian golden hamster (Mesocricetus auratus), is a clinically relevant animal model for multiple human diseases. Hence, the major objectives of this study were to characterize the structure and function of Mesocricetus auratus MIF (MaMIF) and finally evaluate its effect on pancreatic tumor growth in vivo. Initially, the recombinant MaMIF was cloned, expressed and purified in a bacterial expression system. The MaMIF primary sequence, biochemical properties, and crystal structure analysis showed greater similarity with human MIF. The crystal structure of MaMIF illustrates that it forms a homotrimer as known in human and mouse. However, MaMIF exhibits some minor structural variations when compared to human and mouse MIF. The in vitro functional studies show that MaMIF has tautomerase activity and enhances activation and migration of hamster peripheral blood mononuclear cells (PBMCs). Interestingly, injection of MaMIF into HapT1 pancreatic tumor-bearing hamsters significantly enhanced the tumor growth and tumor-associated angiogenesis. Together, the current study shows a structural and functional similarity between the hamster and human MIF. Moreover, it has demonstrated that a high level of circulating MIF originating from non-tumor cells might also promote pancreatic tumor growth in vivo.

Clinically relevant animal models help in understanding the pathogenesis of different human and animal diseases and play crucial roles in developing new therapeutics against them ${ }^{1}$. In spite of the dominance of the mouse as an experimental model animal, the hamster has carved its niche as a potential model animal for studying many diseases and for the evaluation of therapeutic agents. Hamsters are frequently used in various disease pathogenesis studies due to the ease of handling them and the similarity to humans in disease development. Hamsters are important animal models for studying various infectious diseases of humans ${ }^{2-9}$. The hamster models of pancreatic and oral cancer have gained importance in their respective fields ${ }^{10-15}$. Moreover, these animals have also been instrumental in studying metabolic and/or inflammatory diseases like diabetes and pancreatitis ${ }^{16,17}$. Despite the

\footnotetext{
${ }^{1}$ Tumor Microenvironment and Animal Models Lab, Institute of Life Sciences, Bhubaneswar, Odisha, India. ${ }^{2}$ Macromolecular Crystallography Lab, Institute of Life Sciences, Bhubaneswar, Odisha, India. ${ }^{3}$ Regional Centre for Biotechnology, Faridabad, Haryana, India. ${ }^{4}$ Manipal Academy of Higher Education, Manipal, Karnataka, India. ${ }^{5}$ Molecular Biology of Abiotic Stress Lab, Institute of Life Sciences, Bhubaneswar, Odisha, India. ${ }^{6}$ Department of Microbiology, Odisha University of Agriculture and Technology, Bhubaneswar, Odisha, India. ${ }^{7}$ These authors contributed equally: Voddu Suresh, Rajivgandhi Sundaram and Pujarini Dash. *email: dileep@ils.res.in; senapati@ ils.res.in
} 
fact that the hamster is an important clinically relevant animal model for different diseases, it is not used to its full potential. In this aspect, the non-availability of complete genetic information of these animals and lack of biological reagents like recombinant proteins and antibodies related to them are the major constraints.

Macrophage migration inhibitory factor (MIF) is a pro-inflammatory cytokine with pleiotropic functions in various pathophysiological processes ${ }^{18-21}$. Though initially identified as a T cell-derived cytokine that inhibits macrophage migration, its pleiotropic effects on immune cells, cancer cells, as well as non-cancerous cells made MIF more enigmatic to the researchers. Involvement of MIF in several human diseases like pulmonary hypertension, endothelial cell growth, atherosclerosis, wound healing, viral infection, many cancers including lung, colon, prostate, breast and pancreatic cancer has gained significant interest in this molecule ${ }^{22}$. In one of our earlier published study, we have characterized HapT1 cell line-based Syrian golden hamster tumor as a model of pancreatic cancer-associated desmoplasia, an event that plays a key role in human pancreatic cancer progression. In that study, for the first time, proteomics analysis of whole-cell lysate from hamster pancreatic stellate cells (PSCs) showed expression of macrophage migration inhibitory factor (MIF) by the cells ${ }^{11}$. At that particular time, due to the unavailability of information and reagents for Mesocricetus auratus MIF (MaMIF), we were unable to investigate the function of this molecule in the hamster model of pancreatic cancer. Hence, in the current study, our major objective was to characterize the MaMIF protein and evaluate the effect of exogenous MIF on the growth of pancreatic tumor in a syngeneic model of hamster pancreatic cancer.

In the current study, we have successfully purified recombinant MaMIF protein from a bacterial protein expression system. Our analysis showed that like human MIF, MaMIF also forms a trimer in solution. A commercially available MIF antibody raised against human MIF cross-reacts with MaMIF. We resolved the trimeric MaMIF crystal structure at 1.8 A resolution, and the structural analysis showed multiple features in MaMIF to be similar to mouse and human MIF. Further, biochemical and cell culture-based studies using endotoxin-free MaMIF showed it's enzymatic (tautomerase) and immunostimulatory activities, which suggest that the purified protein is biologically active. Importantly, all the biological properties of MaMIF investigated in this study were similar to human MIF. At the end, we have investigated the effect of MaMIF on the growth of HapT1 pancreatic tumor in its syngeneic host. The data clearly shows the pro-tumorigenic effect of MaMIF on the HapT1 pancreatic tumor. Taken together, the data presented in this study have unraveled multiple information regarding MaMIF, and indicate the importance of hamster as a model to investigate questions related to the role of MIF in pancreatic cancer progression.

\section{Materials and Methods}

Recombinant MaMIF expression, purification and Immunoblotting. Syrian golden hamster (Mesocricetus auratus) Mif open reading frame sequence (spanning residues 1-115; Supplementary Fig. 1) was PCR amplified and cloned in between NdeI and XhoI sites of a pET22b + vector with an uncleavable C-terminal hexahistidine tag. The protein was expressed in E. coli BL21 (DE3) cells at an $\mathrm{OD}_{600 \mathrm{~nm}}$ of 0.6 , by induction with $0.5 \mathrm{mM}$ IPTG for 4 hours at $37^{\circ} \mathrm{C}$. Cells from 1 liter culture were pelleted down by centrifugation for $10 \mathrm{~min}$ at $6000 \times \mathrm{g}$ and then suspended in $50 \mathrm{ml}$ of buffer A containing $20 \mathrm{mM}$ Tris- $\mathrm{HCl}(\mathrm{pH} 7.5), 20 \mathrm{mM}$ imidazole, $300 \mathrm{mM} \mathrm{NaCl}, 1 \mathrm{mM} \beta \mathrm{ME}, 1 \mathrm{mM}$ PMSF and one tablet of EDTA-free protease inhibitor cocktail (Sigma). The cells were lysed by sonication and the lysate was clarified by centrifugation at $40,000 \times \mathrm{g}$ for $45 \mathrm{~min}$. Recombinant MaMIF was first captured on a Ni-NTA affinity column (HisTrap FF $5 \mathrm{ml}$, GE Healthcare). Then the column was washed with 15 column volumes of buffer A and eluted with a linear gradient of buffer B (buffer A supplemented with $500 \mathrm{mM}$ imidazole), followed by size-exclusion chromatography using a HiLoad 16/600 Superdex 75 pg column (GE Healthcare) with buffer C containing $20 \mathrm{mM}$ Tris- $\mathrm{HCl}$ (pH 7.5), $150 \mathrm{mM} \mathrm{NaCl}$ and $1 \mathrm{mM}$ DTT. The peak fractions containing MIF were pooled and concentrated to $30 \mathrm{mg} / \mathrm{ml}$ and stored at $-80^{\circ} \mathrm{C}$. The purified protein was analyzed on $18 \%$ SDS-PAGE and stained with Coomassie Brilliant Blue to confirm the purity and to get an estimate of the monomeric molecular mass. To estimate the approximate molecular mass of purified MaMIF in native conformation, i.e. to find whether the protein exists in an oligomeric form, analytical size-exclusion chromatography was performed using buffer C with help of Superdex 75 10/300 GL column which was pre-calibrated with Low molecular weight standards (GE Healthcare). For biochemical experiments, endotoxin was removed from purified MaMIF using Vivaspin endotest tubes (Sartorius Biotech, Germany). Next, the E. coli-derived recombinant MaMIF used in our biochemical experiments was used to perform Western blot to elucidate the cross-reactivity of MIF polyclonal antibody (NBP1-81832; Novus Biologicals) towards MaMIF, since this antibody was raised against a recombinant protein with $100 \%$ identity with human MIF and $89 \%$ identity with MaMIF. In parallel, a non-MIF primary antibody with similar isotype (anti-Bak antibody, Rabbit mAb \#12105, CST) was used to detect the background level of MaMIF detection.

CD spectroscopy. CD spectra of $10 \mu \mathrm{M}$ MaMIF in a buffer containing $20 \mathrm{mM}$ Tris- $\mathrm{HCl}(\mathrm{pH} 7.5)$ and $50 \mathrm{mM}$ $\mathrm{NaCl}$ were recorded using a Chirascan $\mathrm{CD}$ spectroscope (Applied Photophysics). The spectra were collected in triplicate from $260 \mathrm{~nm}$ to $190 \mathrm{~nm}$ at $25^{\circ} \mathrm{C}$ at a bandwidth of $1.0 \mathrm{~nm}$ using a quartz cuvette with $10 \mathrm{~mm}$ path length. All the spectra were averaged and the buffer spectrum was subtracted from those of sample. The estimation of secondary structural elements was done using the BeStSel server ${ }^{23}$. The CD intensities (in millidegrees) are plotted against wavelength.

Multiple sequence alignment and phylogenetic tree analysis. Protein sequences of Syrian golden hamster MIF (UniProtKB: A0A140EDM8), mouse MIF (UniProtKB: P34884) and human MIF (UniProtKB: P14174) were aligned using PRALINE multiple sequence alignment program and residue substitution matrix BLOSUM62 at http://www.ibi.vu.nl/programs/pralinewww/. PRALINE sequence conservation score 0 was given for the least conserved position and 10 for the most conserved position of alignment. The phylogenetic tree was constructed with MEGA 7 software ${ }^{24}$ using the Neighbor-Joining method ${ }^{25}$. The optimal tree with the sum of 
branch length is 0.15113287 and was drawn to scale with branch lengths (below the branches) in the same units as those of the evolutionary distances used to infer the phylogenetic tree. The evolutionary distances were computed using the Poisson method in the units of several residue substitutions per position.

Crystallization and data collection. Recombinant MaMIF $(15 \mathrm{mg} / \mathrm{ml})$ was screened for crystallization at $18^{\circ} \mathrm{C}$ using commercially available screens from Hampton Research with the help of a Formulatrix NT8 crystallization robot in 96-well sitting drop crystallization plates. After 3 days, small and two-dimensional crystals appeared in a few conditions. The sequential micro-seeding approach was adopted for further optimization. Towards this end, crystals grown in Hampton Research PEG/Ion HT F8 condition containing 50\% v/v PEG 400, $200 \mathrm{mM}$ lithium sulfate and $100 \mathrm{mM}$ sodium acetate ( $\mathrm{pH} 7.5)$ were crushed by using the seeding kit (Hampton Research) and used for micro-seeding into the same condition, but in bigger drops with $1 \mu \mathrm{l}$ protein and $1 \mu \mathrm{l}$ condition, using hanging drop vapor diffusion method. After a week, bigger crystals appeared but were still two-dimensional in morphology. These crystals were picked, crushed and used as seed material for fresh crystallization screens following the random micro-seed matrix-screening method ${ }^{26}$ in 96 -well sitting drop plates, with the help of an NT8 crystallization robot. Crystals appeared in several new conditions; however, most of them were still two-dimensional and plate-like in morphology. A condition having $200 \mathrm{mM}$ succinic acid (pH 7.0) and $20 \% \mathrm{w} / \mathrm{v}$ PEG 3350 yielded a bigger and thicker crystal after three weeks. The crystal was flash cooled in liquid nitrogen, after transferring to a solution with the composition of the crystallization condition, supplemented with $20 \%$ ethylene glycol. The diffraction images were recorded on an Eiger X 4M detector (DECTRIS) on beamline ID30A-3 (MASSIF-3) of European Synchrotron Radiation Facility (Grenoble, France). 2000 images were collected with an oscillation of $0.1^{\circ}$, at a wavelength of $0.9677 \AA$.

Data processing, structure determination and refinement. The diffraction images were processed using XDS ${ }^{27}$. The crystal structure of MaMIF was solved by the molecular replacement method with the help of the program Molrep ${ }^{28}$ from the CCP4 suite ${ }^{29}$, using the mouse MIF crystal structure (PDB id: $1 \mathrm{mff}$ ) as the search model. Crystallographic refinement and model building were performed iteratively using Refmac $5^{30}$ and $\operatorname{Coot}^{31}$. The quality of the final model was assessed using the Ramachandran Plot from the programs PROCHECK ${ }^{32}$ and Molprobity ${ }^{33}$. All the figures were prepared using PyMOL (Schrödinger, LLC). The data collection and refinement statistics are provided in Table 1. The structure factors and refined coordinates have been deposited in the PDB, with the accession code 6ice.

Tautomerase assay. MIF from other species has been reported to possess one unusual activity of catalyzing the tautomerization of D-dopachrome and L-dopachrome methyl ester into their corresponding indole derivatives ${ }^{34}$. Tautomerase assay was carried out as described previously ${ }^{35}$. To analyze the tautomerase activity of MaMIF, $4 \mathrm{mM}$ of L-3,4-dihydroxyphenylalanine methyl ester was diluted in $5 \mathrm{ml}$ autoclaved, double-distilled water and an appropriate amount of sodium periodate was added to it to make a final concentration of $8 \mathrm{mM}$. The mixture was incubated on ice for $20 \mathrm{~min}$ in the dark. $300 \mu \mathrm{l}$ from this solution was mixed with $700 \mu \mathrm{l}$ of the assay buffer consisting of $50 \mathrm{mM}$ potassium phosphate and $1 \mathrm{mM}$ EDTA ( $\mathrm{pH}$ 6.0). Increasing concentrations of MIF were added to it and the decrease in absorbance at $475 \mathrm{~nm}$ was recorded after different time points of incubation. To confirm the specificity of MIF tautomerase activity, a similar experiment was conducted with BSA as a control.

Effect of MaMIF on the expression of inflammation-associated genes in hamster peripheral blood mononuclear cells (PBMCs). To check the effect of MIF on the expression status of certain inflammation-associated genes in PBMCs, cells were isolated from hamster blood using the Histopaque gradient as suggested by the manufacturer (Sigma, USA). The isolated PBMCs were cultured in 10\% RPMI media and treated with BSA $(100 \mathrm{ng} / \mathrm{ml})$, MaMIF $(100 \mathrm{ng} / \mathrm{ml})^{36-38}$, Polymyxin B $(30 \mu \mathrm{g} / \mathrm{ml})$, MaMIF + Polymyxin B, ISO-1 ((S, R)-3-(4-hydroxyphenyl)-4, 5-dihydro-5-isoxazole acetic acid methyl ester) $(100 \mu \mathrm{g} / \mathrm{ml})$ or MaMIF + ISO-1 for 4 hours. Then cells were harvested in RLT buffer and processed for RNA isolation as described by the manufacturer (Qiagen, USA). RNA quantification was carried out using Nanodrop followed by cDNA synthesis ${ }^{11}$. The expression levels of Tnf- $\alpha$ Il-6, Il-1 $\beta$ and Vegf, and were analyzed in all the cDNA samples through qPCR, by using gene-specific primers. Polymyxin B was used to neutralize any residual LPS present along with MaMIF protein.

Effect of MaMIF on PBMC migration. Prior approval from the Institutional Animal Ethical Committee (Institute of Life Sciences, Bhubaneswar, India) was taken for all the protocols used in the animal studies. All the methods associated with animal studies were performed according to the Committee for the Purpose of Control and Supervision of Experiments on Animal (CPCSEA), India guidelines. Preparation of hamster peripheral blood mononuclear cells was carried out as previously described ${ }^{39}$. Briefly, normal hamsters having no symptomatic diseases were euthanized and blood was collected through the cardiac puncture in a heparinized tube followed by Histopaque (Sigma, 11191) gradient centrifugation. After gradient centrifugation, the cells present in the buffy coat layer were aspirated and washed twice with $10 \mathrm{ml}$ PBS followed by resuspension in 2 to $3 \mathrm{ml}$ of $0 \%$ FBS containing RPMI media. Cell counting was done through the trypan blue dye exclusion method. To check the effect of recombinant MaMIF on the migration of hamster PBMCs, $3.5 \times 10^{5}$ PBMCs in $0 \%$ FBS containing media $(500 \mu \mathrm{l})$ were seeded in $8 \mu \mathrm{m}$ pore trans-well insert (Millicell Hanging Cell Culture Insert, PET $8 \mu \mathrm{m}, 12$-well, MCEP12H48) and added either BSA (100 ng/ml), MaMIF (100 ng/ml), MaMIF (100 ng/ml) + Polymyxin B (30 $\mu \mathrm{g} / \mathrm{ml})$ or Polymyxin B $(30 \mu \mathrm{g} / \mathrm{ml})$ in $1 \%$ FBS containing media, as chemoattractant $(600 \mu \mathrm{l})$ to the lower chamber. Polymixin B, was used to neutralize any endotoxin contamination, if present in the purified MaMIF ${ }^{40}$. Each experimental condition was carried out in triplicate. After 2 hours of incubation at $37^{\circ} \mathrm{C}$ and $5 \% \mathrm{CO}_{2}$, the cells were fixed in $10 \%$ buffered formalin for $10 \mathrm{~min}$ followed by staining with $0.6 \%$ crystal violet. Cells adhered to the upper surface of the membrane were removed with a cotton swab. Migrated cells at the lower surface of the insert were visualized under an inverted bright field microscope and 5 to 8 random images were captured for 


\begin{tabular}{|c|c|}
\hline Parameter & MaMIF \\
\hline \multicolumn{2}{|l|}{ Data Collection } \\
\hline Beamline & ESRF-MASSIF-3 \\
\hline Detector type & Dectris Eiger X 4M \\
\hline Wavelength $(\AA)$ & 0.9677 \\
\hline Data collection temperature $(\mathrm{K})$ & 100 \\
\hline Space group & $\mathrm{P} 2_{1} 2_{1} 2_{1}$ \\
\hline $\mathrm{a}, \mathrm{b}, \mathrm{c}(\AA)$ & $53.88,55.85,112.16$ \\
\hline$\alpha, \beta, \gamma\left({ }^{\circ}\right)$ & $90,90,90$ \\
\hline Resolution $(\AA)$ & $56.08-1.80(1.84-1.80)$ \\
\hline Rmeas (\%) & $0.118(0.599)$ \\
\hline $\mathrm{I} / \sigma(\mathrm{I})$ & $11.8(3.5)$ \\
\hline $\mathrm{CC}(1 / 2)(\%)$ & $0.997(0.868)$ \\
\hline Total number of reflections & $239343(14289)$ \\
\hline Mosaicity $\left({ }^{\circ}\right)$ & 0.65 \\
\hline Completeness (\%) & $98.5(99.7)$ \\
\hline $\mathrm{R}_{\text {merge }}$ & $0.103(0.525)$ \\
\hline Multiplicity & $7.6(7.8)$ \\
\hline Wilson B-factor $\left(\AA^{2}\right)$ & 6.78 \\
\hline Matthews coefficient $\left(\AA^{3} / \mathrm{Da}\right)$ & 2.14 \\
\hline Solvent content $(\%)$ & 42.46 \\
\hline No. of molecules in ASU & 3 \\
\hline \multicolumn{2}{|l|}{ Refinement } \\
\hline No. of unique reflections & $31590(1840)$ \\
\hline $\mathrm{R}_{\text {work }} / \mathrm{R}_{\text {free }}(\%)$ & $17.6 / 21.3$ \\
\hline No. of protein atoms & 2587 \\
\hline No. of solvent atoms & 196 \\
\hline Mean B-factor $\left(\AA^{2}\right)$ & 15.75 \\
\hline \multicolumn{2}{|l|}{ R.m.s. deviations } \\
\hline Bond lengths $(\AA)$ & 0.020 \\
\hline Bond angles $\left({ }^{\circ}\right)$ & 1.807 \\
\hline \multicolumn{2}{|l|}{ Ramachandran plot (\%) } \\
\hline Preferred region & 99.10 \\
\hline Allowed region & 0.90 \\
\hline Outliers & 0.0 \\
\hline
\end{tabular}

Table 1. Crystal data collection and refinement statistics. Numbers in parentheses correspond to the last resolution shell.

each membrane. The numbers of migrated cells were then expressed as the average of total field per membrane in triplicate for each experimental condition. The gene expression analysis and PBMC migration experiments were repeated minimum of three times in triplicate. Although we noticed slight variations between the experimental values, the resulting pattern was quite similar. The data provided in the manuscript represents data obtained from one set of experiments.

Effect of MaMIF on pancreatic tumor growth in vivo. Syrian golden hamsters (3-4 months old; male) were inoculated subcutaneously with $4 \times 10^{5} \mathrm{HapT} 1$ pancreatic cancer cells. Animals were checked for palpable tumors on a daily basis. After six days from the injection of cancer cells, the tumor-bearing animals were randomized into two groups ( $\mathrm{n}=5$ per group) and treated with MaMIF dissolved in PBS $(1 \mathrm{mg} / \mathrm{kg})$ or PBS intraperitoneally for up to 13 days post-inoculation of tumor cells. Tumor dimensions were measured every day, and tumor volume was calculated by using the formula $\mathrm{V}=\left(\mathrm{W}^{2} \times \mathrm{L}\right) / 2$ for caliper measurements where $\mathrm{V}$ is the tumor volume, $\mathrm{W}$ is the tumor width and $\mathrm{L}$ is the tumor length. All the animals were sacrificed on the $20^{\text {th }}$ day and tumors were harvested. After measuring the tumor weights, the samples were preserved and processed for histopathological analysis.

Histology and immunohistochemistry. HapT1 tumors were dissected and fixed in $10 \%$ neutral buffered formalin at room temperature for 48 hours, and then tumor tissues were dehydrated and embedded in paraffin wax. $5 \mu \mathrm{m}$ thick sections were cut and stained with hematoxylin-eosin. For immunohistochemistry, $5 \mu \mathrm{m}$ paraffin-embedded sections were de-paraffinized in xylene and rehydrated with graded ethanol and deionized water, then boiled in acidic $\mathrm{pH}$ citrate buffer (Vector Laboratories) for $20 \mathrm{~min}$ in a steam cooker. Endogenous peroxidase was blocked with $3 \%$ hydrogen peroxide in methanol for $20 \mathrm{~min}$, and then washed with PBS followed by blocking with horse serum (Vector Lab) for $30 \mathrm{~min}$. Then sections were incubated with the anti-Ki67 
antibody (VP-RM04, Vector Laboratories) overnight at $4{ }^{\circ} \mathrm{C}$ in a humidified chamber followed by washing with PBS. Then slides were incubated for $45 \mathrm{~min}$ at room temperature with a horse anti-rabbit/mouse IgG biotinylated universal antibody (Vector Laboratories) and then with ABC reagent for $30 \mathrm{~min}$. The stain was developed with 3, 3'-diaminobenzidine (DAB; Vector Laboratories) substrate according to the manufacturer's instructions. The slides were counter-stained with hematoxylin, dehydrated, cleared and mounted with mounting media (Vector Laboratories). Slides were observed under a Zeiss ApoTome. 2 microscope and images captured at 10x, 20x and 40x magnifications. For quantification of blood vessels, multiple images from different areas of Haematoxylin \& Eosin (H\&E) stained HapT1 tumor sections were digitally captured and the number of blood vessels/capillaries per field were counted.

Effect of MaMIF on the overall growth of HapT1 cells in vitro. To check the effect of MaMIF on the proliferation of HapT1 cells, 20,000 cells were seeded into the 24-well plate and allowed for attachment. Then, treatment with MaMIF in different concentrations $(0,10,50,100,150,200,300,400 \mathrm{ng} / \mathrm{ml})$ was given in triplicate to each group. After 48 hours, cells were washed twice with PBS and then fixed in $10 \%$ neutral buffered formalin for $10 \mathrm{~min}$ and stained with $0.6 \%$ crystal violet solution for $30 \mathrm{~min}$, followed by de-staining with water. Quantification was done by dissolving crystal violet in $10 \%$ acetic acid for $15 \mathrm{~min}$. Absorbance was measured at $570 \mathrm{~nm}$ using a Varioskan Flash Multimode Reader (Thermo Scientific).

To check the effect of ISO-1 on HapT1 cells growth, 5000 cells/well were seeded in 24-well plates and allowed to adhere for 24 hours at $37^{\circ} \mathrm{C}$ and $5 \% \mathrm{CO}_{2}$. Cells were treated with MaMIF (100 ng/ml), ISO-1 (100 $\left.\mu \mathrm{g} / \mathrm{ml}\right)$ or MaMIF + ISO-1 in triplicate for each group. After 48 hours, MTT $(5 \mathrm{mg} / \mathrm{ml})$ was added to cells and incubated for 4 hours. The media was discarded and all the precipitates were dissolved in $250 \mu$ DMSO to measure the absorbance at $570 \mathrm{~nm}$ using Varioskan Flash Multimode Reader (Thermo Scientific).

Effect of MaMIF on VEGF expression in HapT1 cells. To check the effect of MaMIF on the expression status of VEGF in HapT1 cells, 1,00,000 cells were seeded into $60 \mathrm{~mm}$ petri plate. After cells got attached, treatment was given to different groups such as control, MaMIF $(100 \mathrm{ng} / \mathrm{ml})$, ISO-1 (100 $\mu \mathrm{g} / \mathrm{ml})$ and MaMIF + ISO- 1 . After 48 hours of the treatment, cells were processed and RNA isolated using RNA isolation kit (Qiagen, USA). RNA quantification was done using Nanodrop spectrophotometer (Thermo Scientific) followed by cDNA synthesis. The expression status of Vegf was analyzed with the help of qPCR, by using the following specific primers: Forward: CTGGCTGGGTCACTAACA; Reverse: TTCTGGCTTTGTTCTGACTT.

Knockdown of MaMIF by siRNA transfection. Small interfering RNA (siRNA) oligonucleotides targeting MaMIF were purchased from Eurogentec. The sequence of Sense siRNA was as follows: $5^{\prime}$-UAAUAGUUGAUGUAGA UCCGG-3' and that of the Anti-sense siRNA was 5'-CCGGAUCUACAUCAACUAUUA-3'. 20,000 HapT1 cells were seeded in 24-well plates and cultured for 24 hours with MEM Eagle medium (PAN Biotech). Cells were transfected with the scramble and MIF siRNA having a final concentration of $10 \mathrm{nM}$ using Lipofectamine RNAiMAX transfection reagent (Invitrogen) according to the manufacturer's instructions. After 48 hours of transfection, cells were harvested for qRT-PCR. In parallel, the corresponding culture plates were processed for crystal violet staining and quantification. In vitro viability experiments were performed with minimum of three technical, as well as three experimental replicates and the data shown is an average of the averages $(n=3)$.

\section{Results}

Expression and purification of recombinant MaMIF. Although MIF was the first described cytokine, its role in some important pathophysiological conditions across different species have remained unclear. MIF from human and mouse or even from different human parasites have been structurally and functionally characterized $^{41-43}$. In this study, we cloned and purified MaMIF as described in the materials and methods section. In one of our previous studies, we had cloned MaMIF coding sequence (CDS) from mRNA isolated from hamster pancreatic stellate cells ${ }^{11}$. The mouse genome is known to harbor multiple MIF-related sequences; however, only the true Mif gene has an intron/exon structure and a $5^{\prime}$ untranslated region ${ }^{44}$. Although the mouse MIF-related pseudogenes are highly homologous to cDNA, those contain different mutations that would generate truncated or altered MIF-like proteins. Hence, before cloning the hamster MifCDS into the expression vector, we checked the hamster Mif genome organization. In silico analysis of recently updated genome sequence available in NCBI database (NW_004801628.1) confirmed that the MifCDS cloned in this work is from the true Mif gene, which has a similar genomic organization as the human and mouse genes (Supplementary Fig. 1). The expressed and purified MaMIF showed an approximate molecular mass of $12.5 \mathrm{kDa}$ with more than $95 \%$ purity as observed from $18 \%$ SDS-PAGE, stained with Coomassie Brilliant Blue (Fig. 1A). Comparison of the elution volume of MaMIF with low molecular weight protein standards in the analytical size-exclusion chromatographic experiment suggested the molecular mass of MaMIF in solution to be approximately three-times higher $(39 \mathrm{kDa})$ than the monomer mass $(12.5 \mathrm{kDa})$. This result indicates that the purified recombinant MaMIF exists as a trimer (Fig. 1B), like human and mouse MIF. The CD spectroscopic analysis showed the recombinant protein to be properly folded. The secondary structure content of the protein was estimated to be $27 \% \alpha$-helix, $54 \% \beta$-sheet, $8.8 \%$ turns and $10.2 \%$ unordered structure, with an RMSD and NMRSD values of 0.40 and 0.0237 , respectively, as given in the form of a pie chart along with the profile (Fig. 1C). The Western blot analysis revealed the cross-reactivity of MIF polyclonal antibody (NBP1-81832; Novus Biologicals) towards MaMIF and further confirmed successful expression and purification of the protein (Fig. 1D). Immunoblot analysis using a non-MIF primary antibody with similar isotype (rabbit IgG) as a negative control showed very minimal background signal compared to the MIF-specific primary antibody (Fig. 1E). 
A

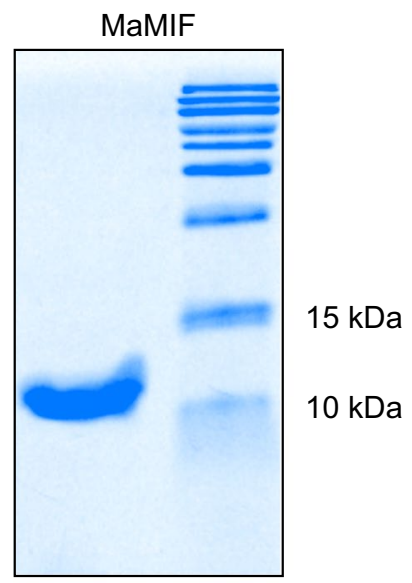

C

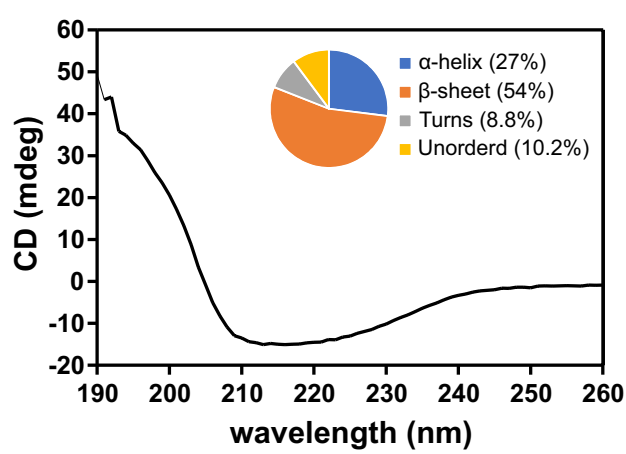

B

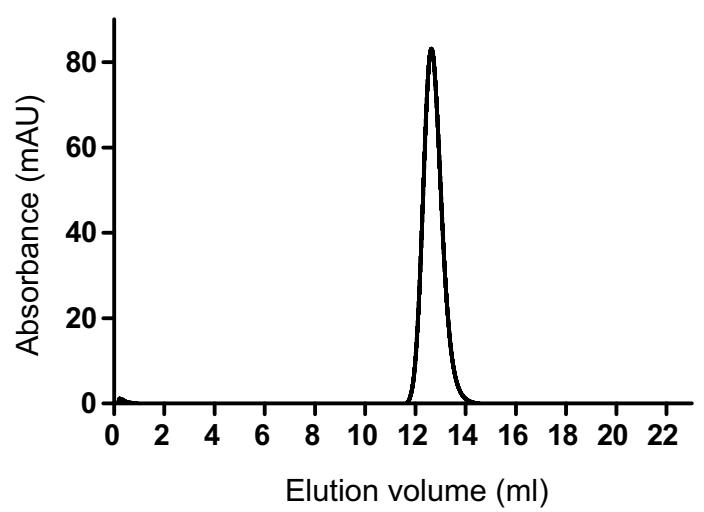

D

E

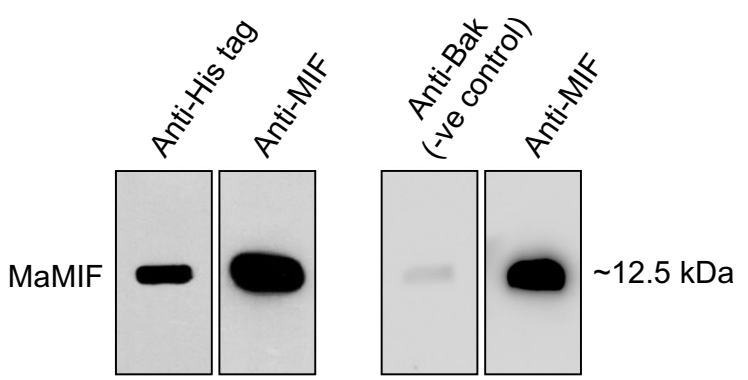

Figure 1. Expression and purification of MaMIF. (A) SDS-PAGE analysis of purified MaMIF suggesting an approximate molecular mass of $\sim 12.5 \mathrm{kDa}$ for the monomer. (B) Superdex $7510 / 300 \mathrm{GL}$ analytical sizeexclusion chromatography profile of MaMIF suggesting an apparent molecular mass of $\sim 39 \mathrm{kDa}$ in solution, corresponding to the size of a trimeric form. (C) CD spectrum of MaMIF confirming a properly folded protein. The pie chart in the inset shows the secondary structure make-up of the protein. (D) Western blot analysis with polyclonal anti-His and anti-MIF antibodies confirming the expressed protein to be MaMIF. (E) Western blot analysis with isotype-matched primary antibody shows the background level of antibody binding to the protein.

Sequence and phylogenetic analysis of MaMIF. Alignment of the primary sequences of MIF from hamster, mouse, and human was performed with PRALINE program. Figure 2A indicates that MaMIF shares $93 \%$ and $89 \%$ sequence identity with mouse MIF and human MIF, respectively. The alignment reveals that the residues responsible for tautomerase activity and substrate binding such as Pro2, Lys33, Ile65, Tyr96, and Asn98 are conserved in MaMIF as well. In addition, the 'CALC' motif required for oxidoreductase activity is also conserved in MaMIF (Fig. 2A). As both the tautomerase and oxidoreductase sites are present in hamster MIF, it might have similar enzymatic and biological activities as that of human and mouse MIF. The phylogenetic analysis of MIF from hamster, mouse and human was carried out using the Neighbor-Joining method with the help of MEGA 7 software further confirmed that MaMIF is evolutionarily closer to the mouse MIF than human MIF (Fig. 2B).

Crystal Structure of MaMIF. The data collection and refinement statistics are given in Table 1. The crystal structure of MaMIF was resolved to $1.8 \AA$ A resolution. It forms a trimer, with three identical molecules in an asymmetric unit. A monomer of MaMIF is made up of six beta strands, four of which form a mixed beta sheet within one subunit, two antiparallel alpha helices and two shorter beta strands on either end of the sheet (Fig. 2C). Also, a very small beta strand $(\beta 7)$ is present towards the $\mathrm{C}$-terminus. The monomer structure is highly conserved among MIF from human, mouse and hamster, as could be observed from the structure alignment (Fig. 2C). The RMSD for the C $\alpha$ backbone atoms between hamster and human MIF (PDB id: 3djh) and between hamster and mouse MIF (PDB id: $1 \mathrm{mf}$ ) are $0.24 \AA$ and $0.26 \AA$, respectively. The trimer adopts a barrel-like architecture formed by three inter-connected monomeric subunits (Fig. 2D), as has been reported for other MIF structures. The trimeric MaMIF structure is retained by the short beta strands $\beta 3$ of one monomer and $\beta 6$ of the third monomer becoming part of the beta sheet formed by the beta strands $\beta 1, \beta 2, \beta 4$ and $\beta 5$ of the second monomer in between, together forming a six-stranded beta sheet. Herein, $\beta 3$ of monomer- 1 comes in close proximity and form hydrogen-bond network, typical of beta sheets, with $\beta 2$ of the monomer- 2 and $\beta 6$ of monomer- 3 comes close to $\beta 5$ of monomer- 2 . This contribution of beta strands by all three monomers to their respective adjacent monomers to form beta 
A

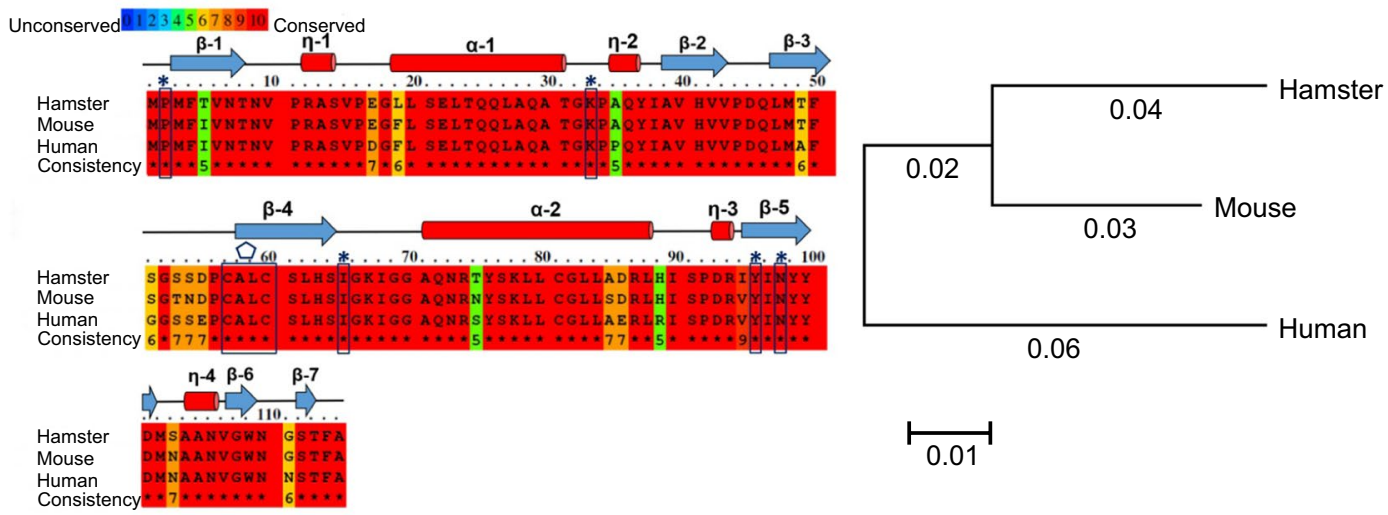

C

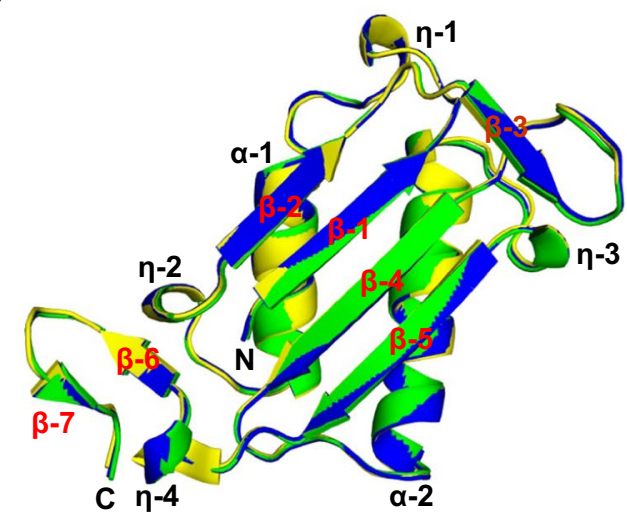

E

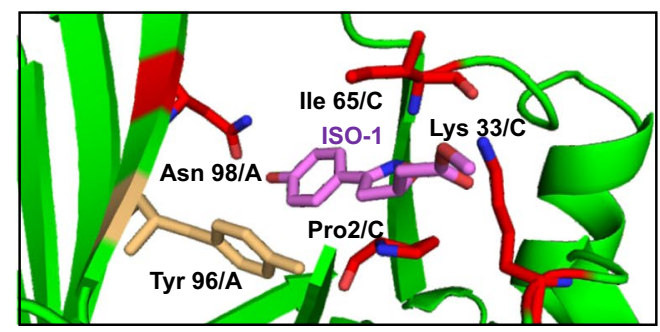

Human

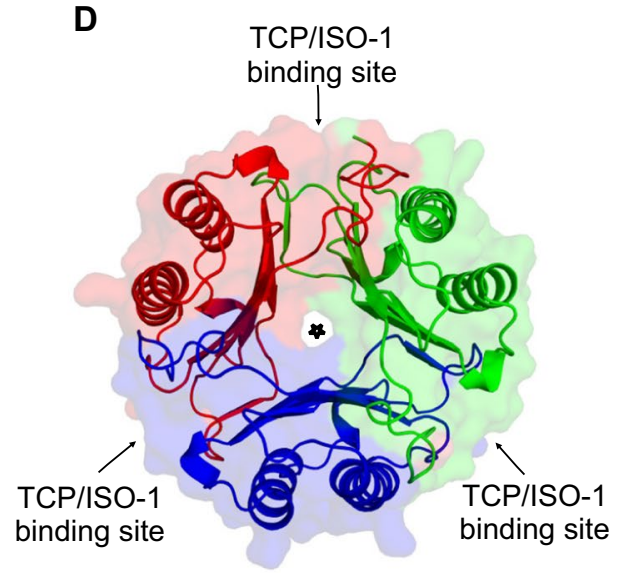

$\mathbf{F}$

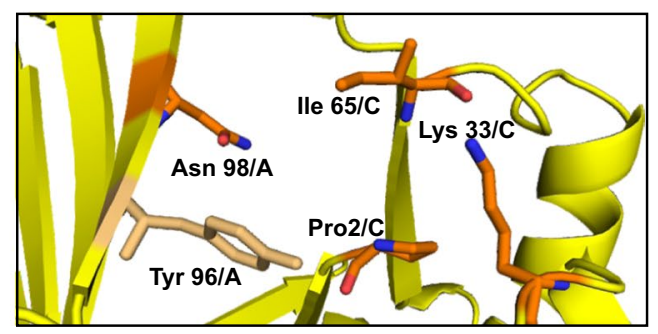

Hamster

Figure 2. Syrian golden hamster MIF protein sequence and structural analysis. (A) Primary sequences of MIF from the Syrian golden hamster (UniProt accession number A0A140EDM8) mouse (P34884) and human (P14174) were aligned with PRALINE program. The conserved residues are shown in red color. The boxes with cap indicate the conserved residues of the tautomerase active site, and the polygon label above the boxed sequence shows the conserved 'CALC' motif responsible for the oxidoreductase activity. Secondary structural elements of MaMIF are indicated above the aligned sequences. (B) Phylogenetic tree of hamster, mouse and human MIF constructed using MEGA 7.0 software. The branch-lengths indicate the evolutionary distances. (C) Superposition of the monomeric structure of MIF from hamster (yellow; PDB id: 6ice, from this work), mouse (blue; PDB id: $1 \mathrm{mfi}$ ) and human (green; PDB id: $3 \mathrm{djh}$ ) in cartoon representation, revealing similar 3D-structure. The MaMIF structure aligns with human MIF and mouse MIF with RMSDs of $0.24 \AA$ and $0.26 \AA$, respectively for the C $\alpha$ atoms. (D) Crystal structure of homo-trimeric MaMIF in cartoon representation, along with semi-transparent surface representation. The monomers are colored in red, green and blue. The three tautomerase catalytic pockets (TCP; also, the sites for ISO-1 binding) located at the monomer-monomer interfaces are labeled with arrows and the central solvent-accessible channel is marked with a star sign. (E) A magnified view of the tautomerase catalytic pocket (TCP) of the ISO-1 bound human MIF structure (PDB id: 1ljt) in green, wherein the active site residues Pro2, Lys33, Ile65, and Asn98 that are interacting with ISO-1 are colored in red and Tyr96 which is part of the catalytic pocket, and bind to ISO- 1 via $\pi$-interaction is colored in light orange; all in stick representation. ISO-1 is shown in stick representation, in magenta color. (F) A similar magnified view as (E) for MaMIF TCP from this work, wherein the structure is shown in yellow, the active site residues Pro2, Lys33, Ile65, and Asn98 are shown in orange and Tyr96 in light orange; all in stick representation. 
sheets seems to provide stability to the trimeric structure. In the trimeric ring structure of MIF, the beta sheets of the three monomers together form a solvent-accessible central water channel, whose role in the context of MIF function is still not known ${ }^{41}$. A MIF trimer has been reported to have three catalytic pockets for tautomerase activity, located in the monomer-monomer interface between the three monomers (Fig. 2D). The residues Pro2, Lys33 and Ile65 from one monomer and the residues Tyr96' and Asn98' from its adjacent monomer ${ }^{45}$, together form the catalytic pocket responsible for the tautomerase activity in human and mouse MIF and these residues are conserved in MaMIF as well. This would explain why MaMIF also shows tautomerase activity (described in the next section). Tautomerase activity of human MIF is known to be inhibited by the MIF-inhibitor ISO- $1^{46}$. ISO-1 binds to the residues Pro2, Lys33, Ile65, Tyr96, and Asn98 ${ }^{47}$, all of which form part of the tautomerase catalytic site located at the interface of two monomers (Fig. 2E, Supplementary Fig. 2). The aromatic ring of human MIF Tyr96 and the hydroxyphenyl ring of ISO-1 make a $\pi$-interaction, wherein, the angle between the ring planes is $78^{\circ}$ and the distance (edge-to-face) between the ring centroids is $4 \AA$. As the residues of the catalytic pocket and ISO-1 binding are completely conserved in MaMIF (Fig. 2F), ISO-1 and other similar compounds binding to the catalytic pocket would block MaMIF catalytic function as well. Several reported MIF structures have sulfate ions bound to the positively charged residues lining the central solvent channel (involved in transporting the negative ions/ligands) or to the peripheral helix of MIF ${ }^{48-50}$. However, the MaMIF structure from this work did not have any sulfate ions, presumably because the final crystallization condition did not have any sulfate.

Tautomerase activity of MaMIF. Unlike all other cytokines, MIF has a tautomerase activity, and it performs this enzymatic function through an $\mathrm{N}$-terminal catalytic proline base $\mathrm{e}^{51}$. In this study, the tautomerase activity of the purified MaMIF was analyzed using L-dopachrome methyl ester substrate. The activity was determined after background correction for the non-enzymatic activity (dopachrome dependent non-specific activity; Supplementary Fig. 3A,B). As the linear range of the kinetic assay for the L-dopachrome methyl ester is too short, the slope value from the initial linear part (ranging from 0 to $15 \mathrm{sec}$.) of the declining curve ( $\Delta$ abs. $\left.\mathrm{s}^{-1}\right)$ was considered to determine the activity (Supplementary Fig. 3C). Supplementary Fig. 3D shows that BSA by itself, which was used as a negative control has no tautomerase activity. However, the addition of MaMIF to the BSA showed enzymatic activity.

The rate of tautomerase activity of the protein (i.e., the amount of the product formed per second) showed a typical enzymatic response, wherein activity increased with increasing concentration of protein at lower concentrations and revealed a saturation tendency at higher concentrations (Fig. 3A). The initial velocities of the reaction were measured as a function of substrate concentration from 0 to $800 \mu \mathrm{M}\left(\mathrm{nmol} \mathrm{ml}^{-1}\right)$. The data presented in Fig. $3 \mathrm{~B}$ was used to determine the $\mathrm{K}_{\mathrm{m}}$ by non-linear least-squares fit method following a simple Michaelis-Menten equation (Fig. 3C). The calculated $\mathrm{K}_{\mathrm{m}}$ value is $665 \mu \mathrm{M}\left(\mathrm{nmol} \mathrm{ml}^{-1}\right)$.

Effect of MaMIF on activation and migration of PBMCs. MIF is known as a potent pro-inflammatory cytokine and regulates activation and migration of immune cells ${ }^{52}$. Exogenous MIF treatment induces expression of various pro-inflammatory cytokines (e.g. TNF- $\alpha$, IL-6, IL-1 $\beta$, and IL-8) and angiogenic factors (e.g. VEGF) in different immune and/or endothelial cells ${ }^{53-55}$. In this study, we investigated the effect of endotoxin-free MaMIF on the activation of hamster PBMCs in the presence and absence of ISO-1. Even though we removed endotoxin using Vivaspin endotest tubes, we further eliminated the chance of any residual endotoxin presence by neutralizing with polymyxin B $(30 \mu \mathrm{g} / \mathrm{ml})^{56}$. As a readout of PBMC activation, the level of Tnf- $\alpha, I l-6, I l-1 \beta$, and Vegf expression was measured through quantitative PCR analysis. Exposure to exogenous MaMIF induced significantly higher expression of Tnf- $\alpha(\mathrm{p}<0.0001), I l-6(\mathrm{p}<0.001)$, and $V e g f(\mathrm{p}<0.0001)$ in PBMCs and the addition of polymyxin B did not alter these effects of MaMIF (Fig. 3D). The tautomerase activity of MIF is known to induce cytokine expression in PBMCs and ISO-1 partially inhibits the MIF induced cytokine expression ${ }^{57}$. Except for Vegf, the MaMIF-induced expression of other factors (Il-1 $\beta, I l-6$, and Tnf- $\alpha$ ) got considerably suppressed upon ISO-1 treatment (Fig. 3D). Interestingly, ISO-1 itself suppressed the endogenous level of Tnf- $\alpha$, Il-1 $\beta$, and Il-6 in PBMCs, which indicates the role of endogenous MIF in regulating the expression of these factors in PBMCs (Fig. 3D). Effect of different cytokines on immune cell activation and migration/recruitment together contribute to the overall inflammation at the site of injury and/or infection. Human MIF is known to act as a chemoattractant for PBMCs ${ }^{52}$. Hence, we wanted to check whether MaMIF has a similar effect on the migration properties of PBMCs. The results of our trans-well migration assay showed a significantly higher number of migrated PBMCs $(\mathrm{p}<0.0001)$ in conditions where MaMIF was used as a chemoattractant as compared to BSA (Fig. 3E).

Effect of MaMIF on the growth of HapT1 pancreatic tumor in its syngeneic host. In pancreatic cancer patients, the tumor tissues and circulating blood have a higher level of MIF than in healthy subjects ${ }^{58,59}$. Pancreatic cancer cells overexpress MIF and are believed to be the major contributor to the overall MIF level in the pancreatic tumor microenvironment. At the same time, multiple normal cells like immune cells, mesenchymal stem cells, endothelial cells, etc. also express MIF ${ }^{60}$. To understand the pathophysiological role of MIF in pancreatic cancer progression, most of the studies have adapted genetic and chemical targeting of MIF expressed by the cancer cells. However, in these approaches, the effect of MIF produced by normal cells on cancer cells (paracrine effects) is not being addressed. Hence, in the current study, we evaluated the impact of MaMIF on the growth of HapT1 tumors in its syngeneic host. The data in Fig. 4A,B shows that systemic administration of MaMIF significantly enhanced HapT1 tumor growth. Though tumor uptake was $100 \%$ in both PBS and MaMIF injected groups, but after 20 days of cancer cells injection, the tumor weight of MaMIF injected tumors was significantly higher $(\mathrm{p}<0.0005)$ than PBS injected tumors (Fig. 4C). Moreover, the presence of a substantially higher number of Ki67-positive cells $(\mathrm{p}<0.0001)$ in the tumors of MaMIF-injected animals than PBS injected animals (Fig. 4D,E) corroborates the observed pro-tumorigenic effect of exogenous MIF on pancreatic tumors in vivo. 
A

B

C
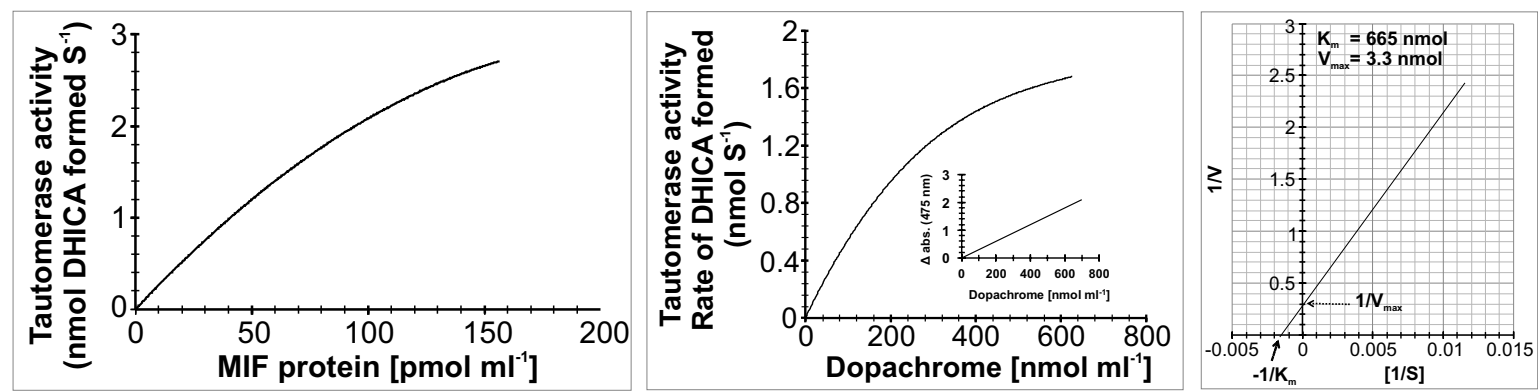

D
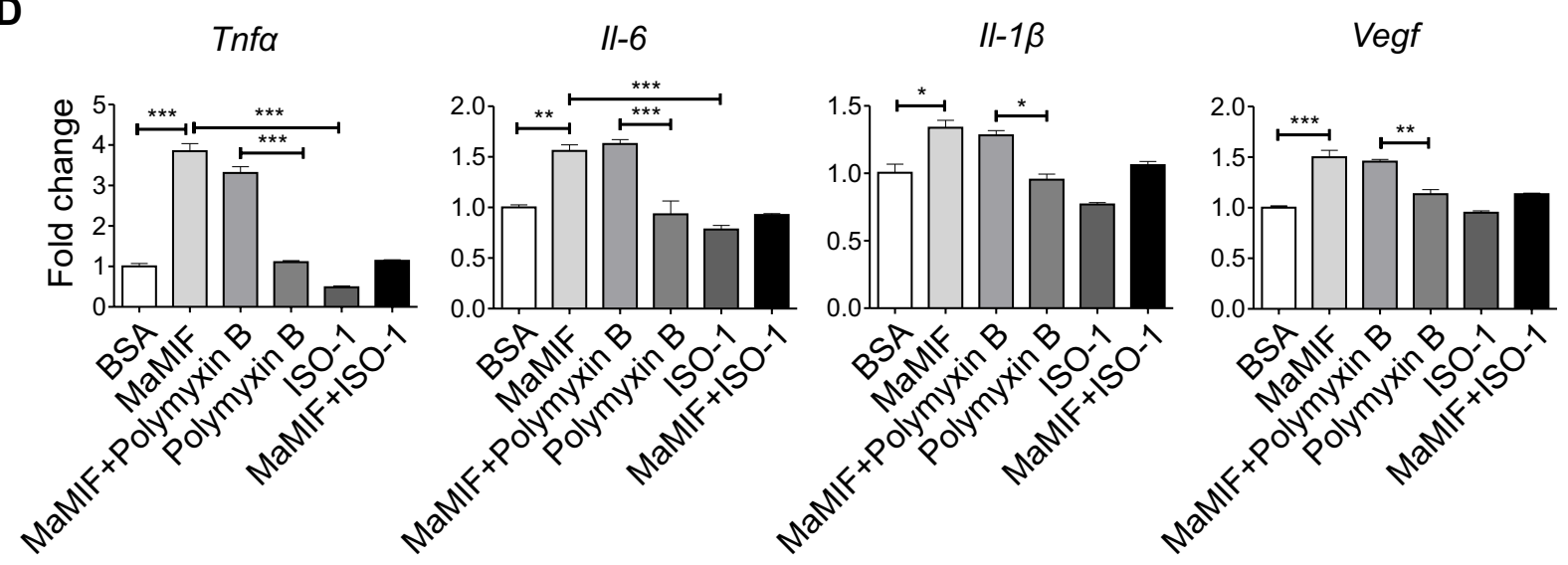

E
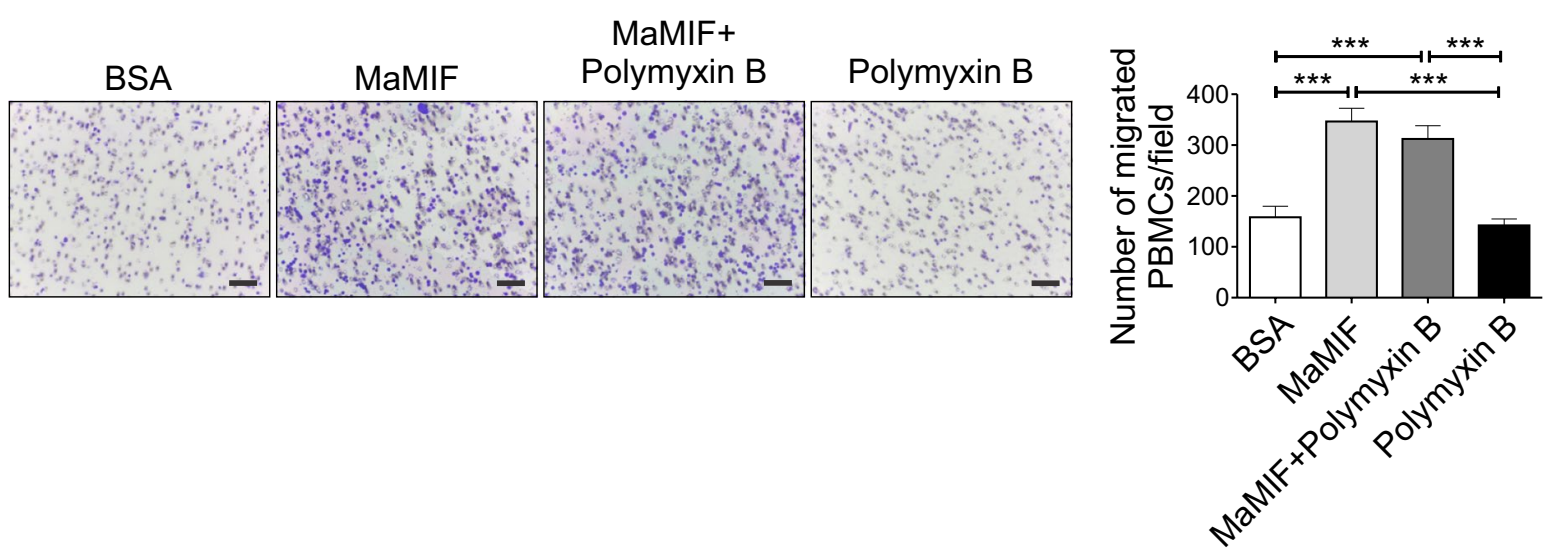

Figure 3. In vitro functional analysis of MaMIF. (A) Data shows the MaMIF-tautomerase activity under the increasing concentration of this protein. (B) Graphical representation showing the influence of increasing concentrations of dopachrome on MIF-tautomerization activity. (C) Determination of the $\mathrm{K}_{\mathrm{m}}$ and $\mathrm{V}_{\max }$ for the substrate-dependent MIF-tautomerase activity. (D) Figure shows the expression of Tnf- $\alpha, I l-6, I l-1 \beta$, and Vegf in PBMCs following stimulation with BSA $(100 \mathrm{ng} / \mathrm{ml})$, MaMIF $(100 \mathrm{ng} / \mathrm{ml})$, Polymyxin B $(30 \mu \mathrm{g} / \mathrm{ml})$, MaMIF + Polymyxin B, ISO-1 $(100 \mu \mathrm{g} / \mathrm{ml})$ and MaMIF + ISO-1 for 4 hours $(\mathrm{n}=3)$. Data represents the mean $\pm \mathrm{SEM}, * \mathrm{p}<0.05 ; * * \mathrm{p}<0.001 ; * * * \mathrm{p}<0.0001$ using one-way ANOVA with Bonferroni's Multiple Comparison Test. (E) Results of the trans-well migration assay show the effect of MaMIF on the migration property of PBMCs. Digital images of the stained trans-well porous membrane show PBMCs (blue colored) that have migrated through the pores after treatment with BSA $(100 \mathrm{ng} / \mathrm{ml})$, MaMIF $(100 \mathrm{ng} / \mathrm{ml})$, Polymyxin B $(30 \mu \mathrm{g} / \mathrm{ml})$, MaMIF + Polymyxin B, ISO-1 $(100 \mu \mathrm{g} / \mathrm{ml})$ and MaMIF + ISO-1 for 2 hours. Quantification of the number of migrated cells in different conditions is shown through the bar graph $(n=3)$. Each bar represents the mean \pm SEM, $* * * \mathrm{p}<0.0001$ using a Student t-test. The scale bar is $100 \mu \mathrm{m}$.

Mechanistic aspects of MaMIF-mediated HapT1 tumor growth in its syngeneic host. Overexpression of MIF by cancer cells is known to promote tumor growth through multiple mechanisms ${ }^{61}$. To check the direct effect of MaMIF on HapT1 cancer cells growth, we treated HapT1 cancer cells with MaMIF in vitro and after 2 days, the number of viable cells was quantified through crystal violet staining of the cultured cells. 
A

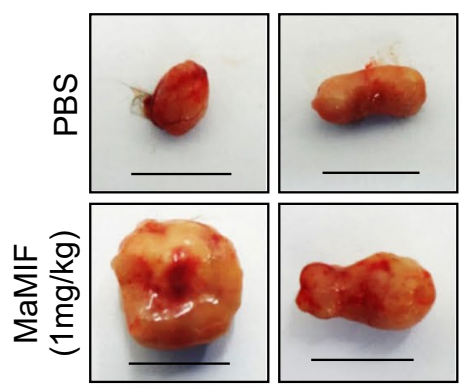

C

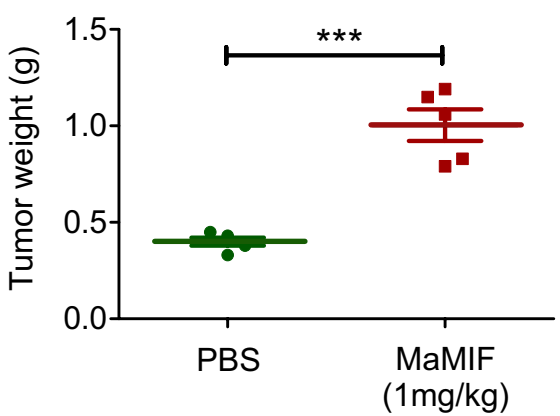

D
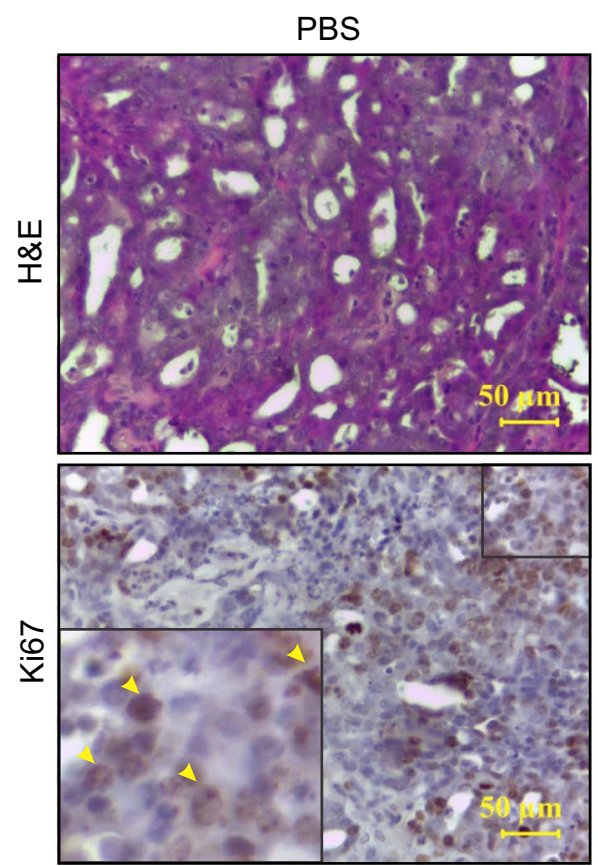

B

$E$
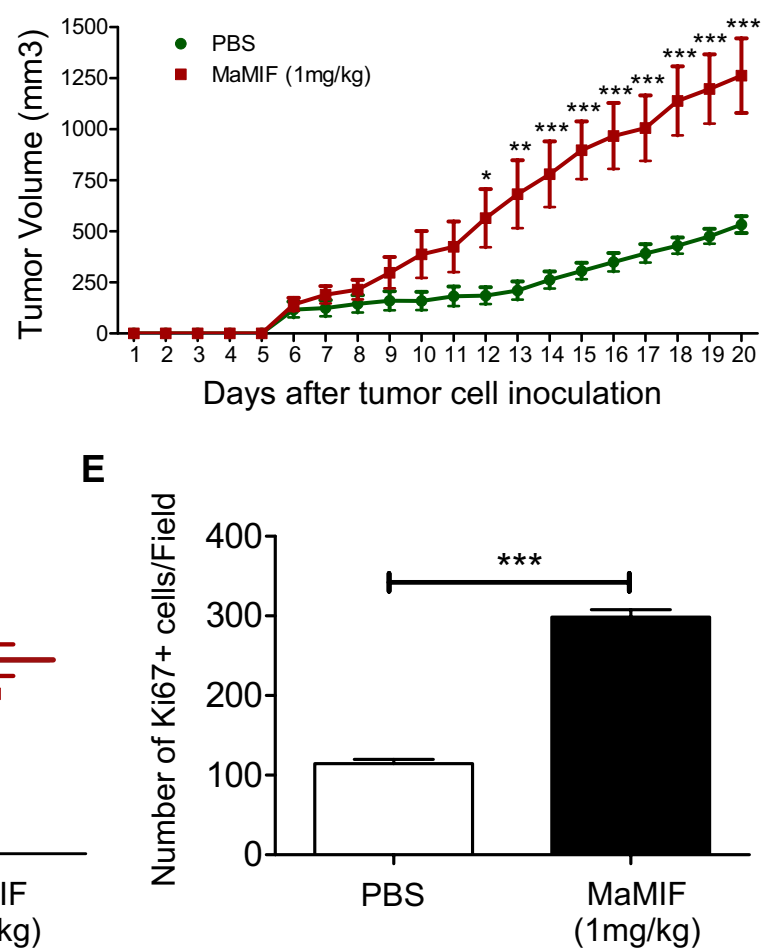

$\operatorname{MaMIF}(1 \mathrm{mg} / \mathrm{kg})$
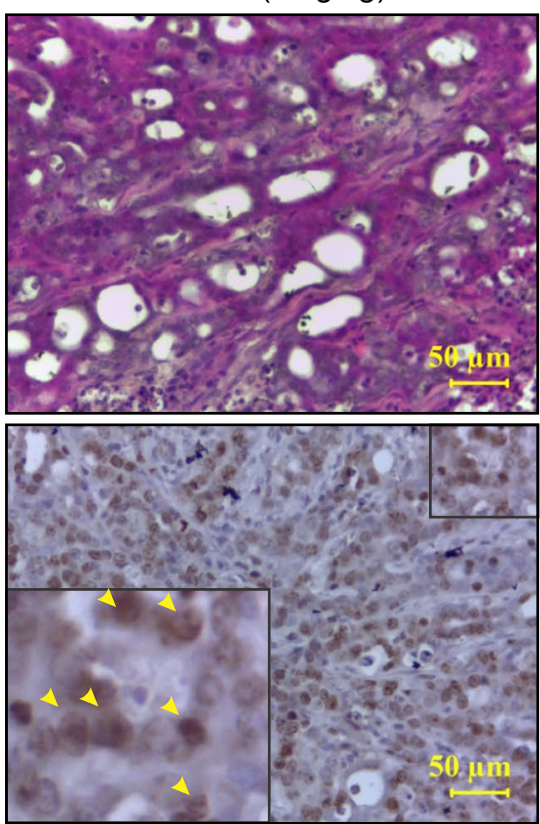

Figure 4. Effect of MaMIF on HapT1 tumor growth in its syngeneic host. (A) Representative digital images of HapT1 tumors obtained from PBS or MaMIF injected animals show bigger tumor mass in MaMIF injected groups. The scale bar is equivalent to $1 \mathrm{~cm}$. (B) Graphical representation of tumor volume calculated every day after initiation of treatment revealed a significant increase in the tumor growth in MaMIF injected animals $(\mathrm{n}=5)$. Data represents the mean \pm SEM, $* * * \mathrm{p}<0.0001$ using two-way ANOVA. (C) The dot-plot graph showing a significant increase in HapT1 tumor weight in MaMIF treated animals than control $(\mathrm{n}=5)$. Data represents the mean \pm SEM, $* * * \mathrm{p}<0.0001$ using a Student $\mathrm{t}$-test. (D) Hematoxylin and Eosin (H\&E) stained HapT1 tumor tissue sections show moderately differentiated tumors in all the conditions (PBS or MaMIF injected). The lower panel representative images show tissue sections stained with Ki67 antibody. Images were captured at $40 \times$ magnification. (E) Graph showing the number of Ki67 positive cells/field $(n=5)$. Data represents the mean \pm SEM, $* * * \mathrm{p}<0.0001$ using a Student t-test. 
A

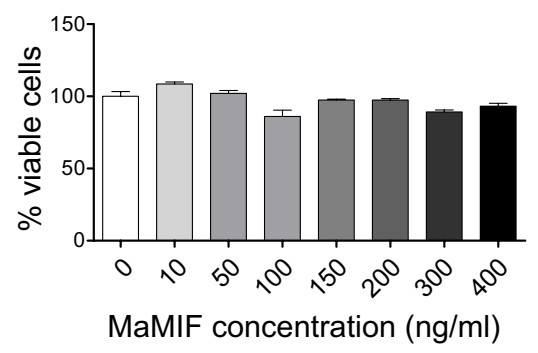

B
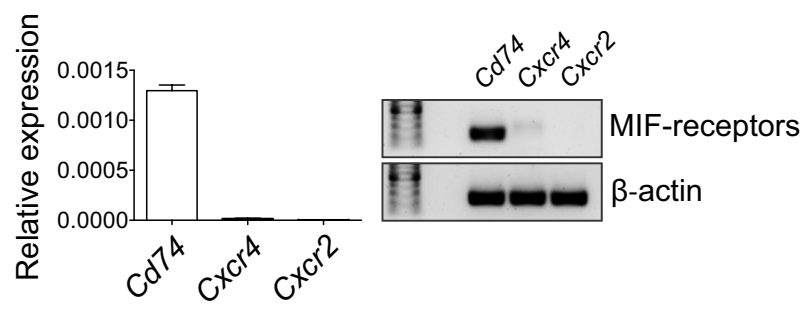

C

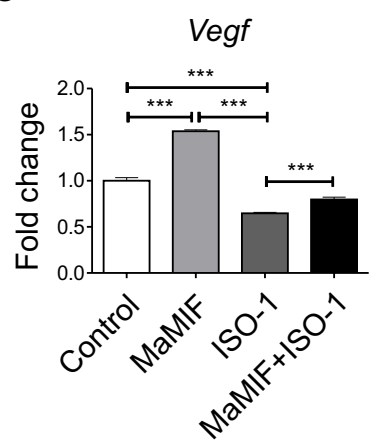

D

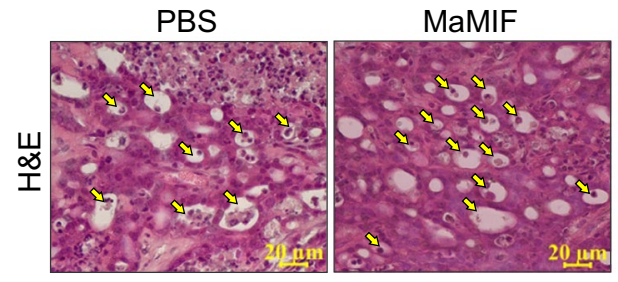

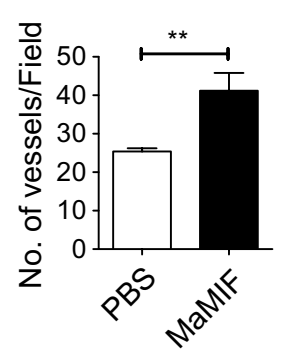

Figure 5. Effect of MaMIF on HapT1 cells growth and angiogenesis. (A) Graph showing the percentage of viable cells upon treatment with different concentrations of MaMIF after 48 hours. (B) Graph showing the relative expression of Cd74, Cxcr4 and Cxcr2 in HapT1 cells and agarose gel image showing the amplified products in qPCR. Relative expression is calculated using formula $2^{-\mathrm{dCT}}, \mathrm{dCT}=\mathrm{CTgene}-\mathrm{CT} \beta$-actin. $(\mathbf{C})$ Graph showing the expression of Vegf in HapT1 cells following stimulation with MaMIF (100 ng/ml), ISO-1 $(100 \mu \mathrm{g} / \mathrm{ml})$ and MaMIF + ISO- 1 for 48 hours $(\mathrm{n}=3)$. Each bar represents the mean \pm SEM. *** $\mathrm{p}<0.0001$ using one-way ANOVA with Bonferroni's Multiple Comparison Test. (D) Representative H\&E images (captured in 40x magnification) showing the number of blood vessels (arrows indicated) in PBS and MaMIF treated tumor tissues. Quantification was done by counting the number of vessels/field in 20x magnification images. Each bar represents the mean \pm the SEM. $* * \mathrm{p}<0.001$ using a Student $\mathrm{t}$-test.

The data showed in Fig. 5A shows that exogenous MaMIF does not have a significant effect on the overall growth of HapT1 cells in vitro. This result prompted us to check the status of MIF receptor expression in HapT1 cells, and our quantitative PCR analysis data clearly shows a high level of Cd74 and low level of Cxcr4, and Cxcr2 expression in HapT1 cells (Fig. 5B). At the molecular level, to check the response of HapT1 cells to exogenously treated MaMIF, we checked the status of Vegf expression in MaMIF-treated and -untreated HapT1 cells. The gene expression analysis showed a significant up-regulation in Vegfexpression upon MaMIF treatment (Fig. 5C). MIF is known to induce tumor angiogenesis in different cancers ${ }^{62}$. Moreover, the up-regulation of Vegf in HapT1 cells upon MaMIF stimulation suggests that MaMIF might affect HapT1 tumor angiogenesis. To check this possibility, HapT1 tumor tissues treated with or without MaMIF were microscopically analyzed and the number of blood vessels was quantified. Quantification of vessel density clearly showed a significantly higher number of blood vessels in MaMIF treated tumors than control tumors (Fig. 5D). Together, the data suggest that circulating MIF of non-tumor origin might promote tumor angiogenesis, thereby promoting overall tumor growth in vivo.

Function of intracellular MIF on the overall growth of HapT1 cells in-vitro. In the aforementioned in vitro experiments, exogenous MaMIF treatment did not show any significant effect on HapT1 cells growth. However, this experiment did not rule out the possible role of intracellular MIF on HapT1 cells. To check the significance of intracellular MIF in HapT1 cells, we first confirmed MIF protein expression in these cells (Fig. 6A). After the confirmation of MIF expression, we depleted MIF expression or inactivated its activity in HapT1 cells by siRNA or ISO-1 treatment, respectively. Further cell viability estimation through crystal violet staining after 48 hours of treatment demonstrated that intracellular MIF depletion or inactivation has a significant effect in reducing the overall growth of these cells in vitro (Fig. 6B-D).

\section{Discussion}

The cross-reactivity of MIF polyclonal antibody (NBP1-81832; Novus Biologicals) generated against a recombinant human MIF having $89 \%$ identity with MaMIF indicates conserved antigenic epitopes in human MIF and MaMIF. Moreover, these results confirm the reliability of using NBP1-81832 antibody to detect the MaMIF. The primary sequence and crystal structure analysis of MaMIF showed that mouse and hamster have an almost similar level of identities with human MIF, which indicates that experiments related to MIF in mouse and hamster 
A

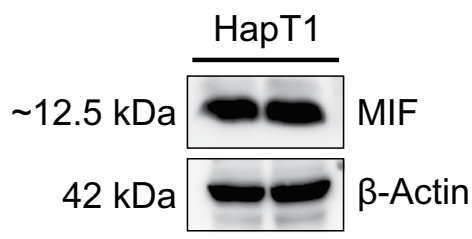

B

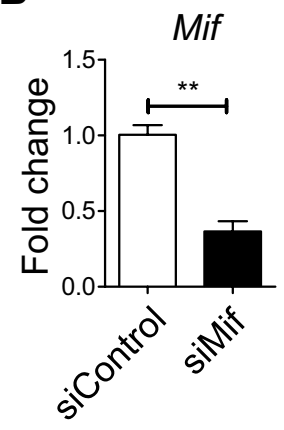

C

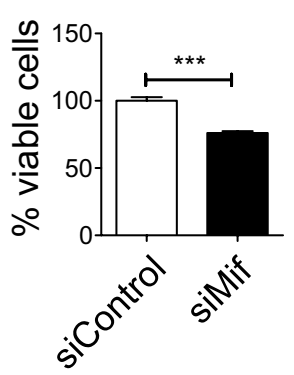

D

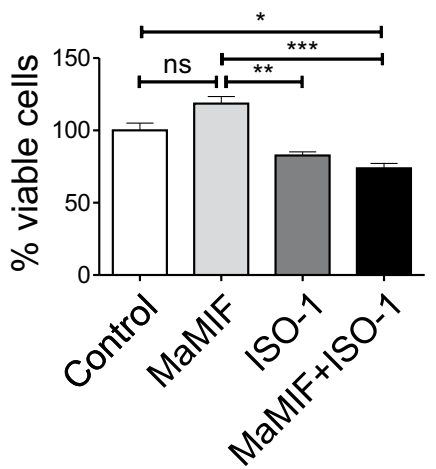

Figure 6. Effect of intracellular MIF on the overall growth of HapT1 cells in-vitro. (A) Immunoblot analysis showing the MIF expressed by the HapT1 cells (Lysates of two different passages). (B) Graph showing the reduced expression of MIF after 48 hours of siRNA transfection $(n=3)$. Each bar represents the mean \pm the SEM. ***p $<0.0001$ using a Student t-test. (C) Graph showing the percentage of viable cells upon siRNA transfection $(n=3)$. Each bar represents the mean \pm the SEM. $* * p<0.001$ using a Student $t-$ test. (D) Graph showing the percentage of viable cells upon treating with MaMIF $(100 \mathrm{ng} / \mathrm{ml})$, ISO-1 $(100 \mu \mathrm{g} / \mathrm{ml})$ and MaMIF + ISO- 1 for 48 hours $(n=3)$. Data represents the mean \pm SEM, $* \mathrm{p}<0.05 ; * \mathrm{p}<0.001 ; * * * \mathrm{p}<0.0001$ using one-way ANOVA with Bonferroni’s Multiple Comparison Test.

might have also similar outcomes. However, it will be apt to mention that the overall outcome of an experimental intervention in any animal model depends on multiple factors like the animal's genetic makeup, physiology, natural behavior, etc. Hence, in certain instances, hamster might be a more clinically relevant model than mice and vice versa. For example, hamster is a good host for multiple infectious agents to which mice are resistant ${ }^{63}$. To investigate the role of MIF in the pathogenesis of these diseases, hamster model might be instrumental. Moreover, conserved structural features like the active site for tautomerase and ISO-1 binding in MaMIF further justifies the suitability and relevance of this model in MIF-related studies (Fig. 2D,E, Supplementary Fig. 2). A study has shown that the diameter of the central channel of MIF from parasites like Giardia lamblia and Plasmodium species is narrower than that of human MIF. This structural difference between human- and parasite-MIF central channels might form a basis for structure-based drug design against the parasites ${ }^{64}$. A wider channel size in human MIF and MaMIF (Fig. 2D) indicates that this structure might have a functional significance in vertebrates, which warrants further investigation.

The $\mathrm{K}_{\mathrm{m}}$ value of MaMIF protein (Fig. 3C) is not in agreement with the MIF proteins of other species (2.4 $\mathrm{mM}^{65} ; 2.7 \mathrm{mM}^{66} ; 2.1 \mathrm{mM}^{67}$ ). This enzyme was found to have much superior activity, with nearly 4 -fold higher sensitivity towards L-dopachrome. This difference might be due to an intrinsic superior enzymatic activity of MaMIF and/or difference associated with the technical process (protein quality, instrument sensitivity, etc.). A further in-depth investigation to compare the tautomerase activity of MaMIF and human MIF, using the same technical procedure might be helpful while exploring for effective MIF-inhibitors. Being a conserved protein and having a unique combination of hormone-, cytokine- and thioredoxin-like properties, MIF is considered as a potent cytokine with pleiotropic effects on immune and inflammatory events ${ }^{68}$. It is known to regulate the expression of pro-inflammatory mediators leading to early patient death in sepsis ${ }^{69,70}$. At the same time, human MIF is known to have a differential effect on the migratory properties of various cell types ${ }^{52}$. The recruitment of immune cells to the site of infection leads to clearance of viral, bacterial and fungal infection but on the other hand, monocytes also contribute to their pathogenesis and inflammation ${ }^{71}$. Effect of MaMIF in augmenting the directional migration of hamster PBMCs towards it shows its chemo-attractant property similar to human MIF (Fig. 3E) ${ }^{52}$. Moreover, the effect of ISO-1 in inhibiting the MaMIF mediated upregulation of the expression of proinflammatory molecules and migration of hamster PBMCs corroborates the information obtained from the structural analysis of MaMIF. These data further suggest that ISO-1 could be reliably used as a MaMIF inhibitor in various in vitro and in vivo studies.

Due to its remarkable involvement in cytokine cascade in the tumor microenvironment, MIF is described as the connecting link bridging cancer with inflammation ${ }^{72}$ and it is also known to be implicated in angiogenesis in multiple cancer types ${ }^{73-75}$. In the recent past, studies have demonstrated the presence of a high level of MIF in different cancer patients. A high level of MIF is reported from the tumor tissues and circulating blood of pancreatic ductal adenocarcinoma (PDAC) patient $5^{58,59}$. The expression level of MIF correlates with poor prognosis of PDAC patients. In most of the studies trying to understand the role of MIF in the progression of different cancers, MIF expression was knocked down in overexpressing cancer cells. However, these attempts do not address the contribution of MIF originating from stromal cells of cancer patients. To best of our knowledge, in the pursuit of understanding the functional role of MIF in pancreatic cancer progression, the current study is the first attempt in which exogenous recombinant MIF has been used to induce high circulating MIF level in pancreatic cancer tumor-bearing animals. The results of this study suggest that MIF of non-cancer cell origin can also promote pancreatic tumor growth. Although HapT1 cells express MIF receptors, the addition of exogenous MIF showing no significant effect on HapT1 cells growth in vitro indicates that these cells are less dependent 
on MIF in a growth-factor enriched culture condition (Fig. 5A,B). A similar kind of effect of MIF has also been reported in mouse $4 \mathrm{~T} 1$ breast tumor cell line ${ }^{61}$. On the other hand, overexpression of Vegf in HapT1 cells upon stimulation with MaMIF and presence of more number of blood vessels in HapT1 tumors suggest a possible mechanism through which circulating MIF might indirectly enhance HapT1 tumor growth by promoting angiogenesis in vivo. At the same time, results shown in Fig. 6 suggest that the intracellular MIF in HapT1 cells has a pro-tumorigenic role. This could be due to its interaction with proteins like p53 and Jab1/CSN5 as having been reported earlier ${ }^{76,77}$. Together, the effect of MaMIF in promoting the growth of HapT1 tumors corroborates and confirms the pro-tumorigenic role of MIF in pancreatic cancer progression. Hence, while designing MIF-targeted therapy in pancreatic cancer, all the possible sources of MIF that can affect overall tumor growth need to be taken into consideration.

Multiple studies have used a MIF concentration of $100 \mathrm{ng} / \mathrm{ml}$ in different cell culture-based studies ${ }^{36}$. However, MIF expression in various disease conditions is variable and dynamic as MIF is a pathophysiologically inducible factor. The major objective of the current study was to check how MaMIF affects different cell types (immune or cancer) and compare it with the already reported properties of human MIF. Our findings might encourage other investigators to explore the clinical relevance of MaMIF in different contexts by designing studies with different relevant MIF concentrations. Moreover, a better understanding of the mechanisms through which circulating MIF can promote pancreatic tumor progression might provide a rationale to design an effective therapeutic strategy against this deadly disease.

\section{Conclusion}

In the current study, MaMIF has been cloned, expressed and purified for the first time, which will be of significant value for future studies. The crystal structure of MIF will provide direction to study the binding affinity of drugs or inhibitors for therapeutic targeting, which will facilitate experiments on the functional role of MIF in different pathological conditions in a hamster model. As the results of this study show MaMIF to be active both enzymatically and biologically, we believe this work will lead to improved strategies for understanding this molecule in the context of different diseases, including cancer in a hamster model. Importantly, the current study provides convincing experimental evidence which suggests that the hamster model of pancreatic cancer can be reliably used to investigate MIF related questions in pancreatic cancer.

Received: 8 March 2019; Accepted: 7 October 2019;

Published online: 29 October 2019

\section{References}

1. Vandamme, T. F. Use of rodents as models of human diseases. Journal of pharmacy \& bioallied sciences 6, 2-9, https://doi. org/10.4103/0975-7406.124301 (2014).

2. Niklasson, B. S., Meadors, G. F. \& Peters, C. J. Active and passive immunization against Rift Valley fever virus infection in Syrian hamsters. Acta pathologica, microbiologica, et immunologica Scandinavica. Section C, Immunology 92, 197-200 (1984).

3. Milazzo, M. L., Eyzaguirre, E. J., Molina, C. P. \& Fulhorst, C. F. Maporal viral infection in the Syrian golden hamster: a model of hantavirus pulmonary syndrome. The Journal of infectious diseases 186, 1390-1395, https://doi.org/10.1086/344735 (2002).

4. Fisher, A. F. et al. Induction of severe disease in hamsters by two sandfly fever group viruses, Punta toro and Gabek Forest (Phlebovirus, Bunyaviridae), similar to that caused by Rift Valley fever virus. The American journal of tropical medicine and hygiene 69, 269-276 (2003).

5. Tesh, R. B. et al. Experimental yellow fever virus infection in the Golden Hamster (Mesocricetus auratus). I. Virologic, biochemical, and immunologic studies. The Journal of infectious diseases 183, 1431-1436, https://doi.org/10.1086/320199 (2001).

6. Xiao, S. Y., Guzman, H., Zhang, H., Travassos da Rosa, A. P. \& Tesh, R. B. West Nile virus infection in the golden hamster (Mesocricetus auratus): a model for West Nile encephalitis. Emerging infectious diseases 7, 714-721, https://doi.org/10.3201/ eid0704.010420 (2001).

7. Wong, K. T. et al. A golden hamster model for human acute Nipah virus infection. The American journal of pathology 163, 2127-2137, https://doi.org/10.1016/s0002-9440(10)63569-9 (2003).

8. Roberts, A. et al. Severe acute respiratory syndrome coronavirus infection of golden Syrian hamsters. Journal of virology 79, 503-511, https://doi.org/10.1128/jvi.79.1.503-511.2005 (2005).

9. Bosco-Lauth, A. M., Han, S., Hartwig, A. \& Bowen, R. A. Development of a Hamster Model for Chikungunya Virus Infection and Pathogenesis. PloS one 10, e0130150, https://doi.org/10.1371/journal.pone.0130150 (2015).

10. Pour, P. M. et al. Current knowledge of pancreatic carcinogenesis in the hamster and its relevance to the human disease. Cancer 47 , 1573-1589 (1981).

11. Suklabaidya, S. et al. Characterization and use of HapT1-derived homologous tumors as a preclinical model to evaluate therapeutic efficacy of drugs against pancreatic tumor desmoplasia. Oncotarget 7, 41825-41842, https://doi.org/10.18632/oncotarget.9729 (2016).

12. Ramachandhiran, D., Vinothkumar, V. \& Babukumar, S. Paeonol exhibits anti-tumor effects by apoptotic and anti-inflammatory activities in 7,12-dimethylbenz(a)anthracene induced oral carcinogenesis. Biotechnic \& histochemistry: official publication of the Biological Stain Commission, 1-16, https://doi.org/10.1080/10520295.2018.1493221 (2018).

13. Pal, R., Villarreal, P., Qiu, S. \& Vargas, G. In-vivo topical mucosal delivery of a fluorescent deoxy-glucose delineates neoplasia from normal in a preclinical model of oral epithelial neoplasia. Scientific reports 8, 9760, https://doi.org/10.1038/s41598-018-28014-8 (2018).

14. Salzwedel, A. O. et al. Combination of interferon-expressing oncolytic adenovirus with chemotherapy and radiation is highly synergistic in hamster model of pancreatic cancer. Oncotarget 9, 18041-18052, https://doi.org/10.18632/oncotarget.24710 (2018).

15. Benali, N. et al. Inhibition of growth and metastatic progression of pancreatic carcinoma in hamster after somatostatin receptor subtype 2 (sst2) gene expression and administration of cytotoxic somatostatin analog AN-238. Proceedings of the National Academy of Sciences of the United States of America 97, 9180-9185, https://doi.org/10.1073/pnas.130196697 (2000).

16. Wang, Y. et al. Experimental Models in Syrian Golden Hamster Replicate Human Acute Pancreatitis. Scientific reports 6, 28014, https://doi.org/10.1038/srep28014 (2016).

17. Liu, C. et al. Effects of berberine on amelioration of hyperglycemia and oxidative stress in high glucose and high fat diet-induced diabetic hamsters in vivo. BioMed research international 2015, 313808, https://doi.org/10.1155/2015/313808 (2015).

18. Bifulco, C., McDaniel, K., Leng, L. \& Bucala, R. Tumor growth-promoting properties of macrophage migration inhibitory factor. Current pharmaceutical design 14, 3790-3801 (2008). 
19. Santos, L. L. \& Morand, E. F. Macrophage migration inhibitory factor: a key cytokine in RA, SLE and atherosclerosis. Clinica chimica acta; international journal of clinical chemistry 399, 1-7, https://doi.org/10.1016/j.cca.2008.09.014 (2009).

20. Kleemann, R. \& Bucala, R. Macrophage migration inhibitory factor: critical role in obesity, insulin resistance, and associated comorbidities. Mediators of inflammation 2010, 610479, https://doi.org/10.1155/2010/610479 (2010).

21. Gilliver, S. C., Emmerson, E., Bernhagen, J. \& Hardman, M. J. MIF: a key player in cutaneous biology and wound healing. Experimental dermatology 20, 1-6, https://doi.org/10.1111/j.1600-0625.2010.01194.x (2011).

22. Babu, S. N., Chetal, G. \& Kumar, S. Macrophage migration inhibitory factor: a potential marker for cancer diagnosis and therapy. Asian Pacific journal of cancer prevention: APJCP 13,1737-1744 (2012).

23. Micsonai, A. et al. BeStSel: a web server for accurate protein secondary structure prediction and fold recognition from the circular dichroism spectra. Nucleic acids research 46, W315-W322, https://doi.org/10.1093/nar/gky497 (2018).

24. Kumar, S., Stecher, G. \& Tamura, K. MEGA7: Molecular Evolutionary Genetics Analysis Version 7.0 for Bigger Datasets. Molecular biology and evolution 33, 1870-1874, https://doi.org/10.1093/molbev/msw054 (2016).

25. Saitou, N. \& Nei, M. The neighbor-joining method: a new method for reconstructing phylogenetic trees. Molecular biology and evolution 4, 406-425, https://doi.org/10.1093/oxfordjournals.molbev.a040454 (1987).

26. Till, M. et al. Improving the success rate of protein crystallization by random microseed matrix screening. Journal of visualized experiments: JoVE, https://doi.org/10.3791/50548 (2013).

27. Kabsch, W. XDS. Acta crystallographica. Section D, Biological crystallography 66, 125-132, https://doi.org/10.1107/s0907444909047337 (2010).

28. Vagin, A. \& Teplyakov, A. Molecular replacement with MOLREP. Acta crystallographica. Section D, Biological crystallography 66, 22-25, https://doi.org/10.1107/s0907444909042589 (2010)

29. The CCP4 suite: programs for protein crystallography. Acta crystallographica. Section D, Biological crystallography 50, 760-763, https://doi.org/10.1107/s0907444994003112 (1994).

30. Murshudov, G. N. et al. REFMAC5 for the refinement of macromolecular crystal structures. Acta crystallographica. Section D, Biological crystallography 67, 355-367, https://doi.org/10.1107/s0907444911001314 (2011).

31. Emsley, P. \& Cowtan, K. Coot: model-building tools for molecular graphics. Acta crystallographica. Section D, Biological crystallography 60, 2126-2132, https://doi.org/10.1107/s0907444904019158 (2004).

32. Laskowski, R. A., MacArthur, M. W., Moss, D. S. \& Thornton, J. M. PROCHECK: a program to check the stereochemical quality of protein structures. Journal of Applied Crystallography 26, 283-291, https://doi.org/10.1107/S0021889892009944 (1993).

33. Chen, V. B. et al. MolProbity: all-atom structure validation for macromolecular crystallography. Acta crystallographica. Section D, Biological crystallography 66, 12-21, https://doi.org/10.1107/s0907444909042073 (2010).

34. Senter, P. D. et al. Inhibition of macrophage migration inhibitory factor (MIF) tautomerase and biological activities by acetaminophen metabolites. Proceedings of the National Academy of Sciences of the United States of America 99, 144-149, https://doi. org/10.1073/pnas.011569399 (2002).

35. Dios, A. et al. Inhibition of MIF bioactivity by rational design of pharmacological inhibitors of MIF tautomerase activity. Journal of medicinal chemistry $45,2410-2416$ (2002).

36. Abdul-Aziz, A. M. et al. MIF-Induced Stromal PKCbeta/IL8 Is Essential in Human Acute Myeloid Leukemia. Cancer research 77, 303-311, https://doi.org/10.1158/0008-5472.can-16-1095 (2017).

37. Le Hiress, M. et al. Proinflammatory Signature of the Dysfunctional Endothelium in Pulmonary Hypertension. Role of the Macrophage Migration Inhibitory Factor/CD74 Complex. American journal of respiratory and critical care medicine 192, 983-997, https://doi.org/10.1164/rccm.201402-0322OC (2015).

38. Alam, A. et al. Novel anti-inflammatory activity of epoxyazadiradione against macrophage migration inhibitory factor: inhibition of tautomerase and proinflammatory activities of macrophage migration inhibitory factor. The Journal of biological chemistry $\mathbf{2 8 7}$, 24844-24861, https://doi.org/10.1074/jbc.M112.341321 (2012).

39. Mookerjee, A., Sen, P. C. \& Ghose, A. C. Immunosuppression in hamsters with progressive visceral leishmaniasis is associated with an impairment of protein kinase $\mathrm{C}$ activity in their lymphocytes that can be partially reversed by okadaic acid or anti-transforming growth factor beta antibody. Infection and immunity 71, 2439-2446 (2003).

40. Cardoso, L. S. et al. Polymyxin B as inhibitor of LPS contamination of Schistosoma mansoni recombinant proteins in human cytokine analysis. Microbial cell factories 6, 1, https://doi.org/10.1186/1475-2859-6-1 (2007).

41. Sun, H. W., Bernhagen, J., Bucala, R. \& Lolis, E. Crystal structure at 2.6-A resolution of human macrophage migration inhibitory factor. Proceedings of the National Academy of Sciences of the United States of America 93, 5191-5196 (1996).

42. Mitchell, R. et al. Cloning and characterization of the gene for mouse macrophage migration inhibitory factor (MIF). Journal of immunology (Baltimore, Md.: 1950) 154, 3863-3870 (1995).

43. Dobson, S. E. et al. The crystal structures of macrophage migration inhibitory factor from Plasmodium falciparum and Plasmodium berghei. Protein science: a publication of the Protein Society 18, 2578-2591, https://doi.org/10.1002/pro.263 (2009).

44. Bozza, M. et al. Structural characterization and chromosomal location of the mouse macrophage migration inhibitory factor gene and pseudogenes. Genomics 27, 412-419, https://doi.org/10.1006/geno.1995.1071 (1995).

45. Lubetsky, J. B., Swope, M., Dealwis, C., Blake, P. \& Lolis, E. Pro-1 of macrophage migration inhibitory factor functions as a catalytic base in the phenylpyruvate tautomerase activity. Biochemistry 38, 7346-7354, https://doi.org/10.1021/bi990306m (1999).

46. Al-Abed, Y. \& VanPatten, S. MIF as a disease target: ISO-1 as a proof-of-concept therapeutic. Future medicinal chemistry 3, 45-63, https://doi.org/10.4155/fmc.10.281 (2011).

47. Lubetsky, J. B. et al. The tautomerase active site of macrophage migration inhibitory factor is a potential target for discovery of novel anti-inflammatory agents. The Journal of biological chemistry 277, 24976-24982, https://doi.org/10.1074/jbc.M203220200 (2002).

48. Sommerville, C. et al. Biochemical and immunological characterization of Toxoplasma gondii macrophage migration inhibitory factor. The Journal of biological chemistry 288, 12733-12741, https://doi.org/10.1074/jbc.M112.419911 (2013).

49. Crichlow, G. V. et al. Alternative chemical modifications reverse the binding orientation of a pharmacophore scaffold in the active site of macrophage migration inhibitory factor. The Journal of biological chemistry 282, 23089-23095, https://doi.org/10.1074/jbc. M701825200 (2007).

50. El-Turk, F. et al. Characterization of molecular determinants of the conformational stability of macrophage migration inhibitory factor: leucine 46 hydrophobic pocket. PloS one 7, e45024, https://doi.org/10.1371/journal.pone.0045024 (2012).

51. Kudrin, A. et al. Human macrophage migration inhibitory factor: a proven immunomodulatory cytokine? The Journal of biological chemistry 281, 29641-29651, https://doi.org/10.1074/jbc.M601103200 (2006).

52. Rajasekaran, D. et al. Macrophage Migration Inhibitory Factor-CXCR4 Receptor Interactions: Evidence for Partial Allosteric Agonism in Comparison with CXCL12 Chemokine. The Journal of biological chemistry 291, 15881-15895, https://doi.org/10.1074/ jbc.M116.717751 (2016).

53. White, D. A. et al. Pro-inflammatory action of MIF in acute myocardial infarction via activation of peripheral blood mononuclear cells. PloS one 8, e76206, https://doi.org/10.1371/journal.pone.0076206 (2013).

54. Calandra, T. et al. MIF as a glucocorticoid-induced modulator of cytokine production. Nature 377, 68-71, https://doi. org/10.1038/377068a0 (1995).

55. Amin, M. A. et al. Migration inhibitory factor up-regulates vascular cell adhesion molecule- 1 and intercellular adhesion molecule- 1 via Src, PI3 kinase, and NFkappaB. Blood 107, 2252-2261, https://doi.org/10.1182/blood-2005-05-2011 (2006). 
56. Stavitsky, A. B. \& Xianli, J. In vitro and in vivo regulation by macrophage migration inhibitory factor (MIF) of expression of MHC-II, costimulatory, adhesion, receptor, and cytokine molecules. Cellular immunology 217, 95-104, https://doi.org/10.1016/s00088749(02)00516-6 (2002).

57. De la Cruz-Mosso, U. et al. MIF promotes a differential Th1/Th2/Th17 inflammatory response in human primary cell cultures: Predominance of Th17 cytokine profile in PBMC from healthy subjects and increase of IL-6 and TNF-alpha in PBMC from active SLE patients. Cellular immunology 324, 42-49, https://doi.org/10.1016/j.cellimm.2017.12.010 (2018).

58. Costa-Silva, B. et al. Pancreatic cancer exosomes initiate pre-metastatic niche formation in the liver. Nature cell biology 17, 816-826, https://doi.org/10.1038/ncb3169 (2015).

59. Yang, S. et al. A Novel MIF Signaling Pathway Drives the Malignant Character of Pancreatic Cancer by Targeting NR3C2. Cancer research 76, 3838-3850, https://doi.org/10.1158/0008-5472.can-15-2841 (2016).

60. Richard, V. et al. Involvement of macrophage migration inhibitory factor and its receptor (CD74) in human breast cancer. Oncology reports 32, 523-529, https://doi.org/10.3892/or.2014.3272 (2014).

61. Simpson, K. D., Templeton, D. J. \& Cross, J. V. Macrophage migration inhibitory factor promotes tumor growth and metastasis by inducing myeloid-derived suppressor cells in the tumor microenvironment. Journal of immunology (Baltimore, Md.: 1950) 189, 5533-5540, https://doi.org/10.4049/jimmunol.1201161 (2012).

62. Chesney, J. A. \& Mitchell, R. A. 25 Years On: A Retrospective on Migration Inhibitory Factor in Tumor Angiogenesis. Molecular medicine (Cambridge, Mass.) 21(Suppl 1), S19-24, https://doi.org/10.2119/molmed.2015.00055 (2015).

63. Wold, W. S. \& Toth, K. Chapter three-Syrian hamster as an animal model to study oncolytic adenoviruses and to evaluate the efficacy of antiviral compounds. Advances in cancer research 115, 69-92, https://doi.org/10.1016/b978-0-12-398342-8.00003-3 (2012).

64. Buchko, G. W. et al. Crystal structure of a macrophage migration inhibitory factor from Giardia lamblia. Journal of structural and functional genomics 14, 47-57, https://doi.org/10.1007/s10969-013-9155-9 (2013).

65. Rosengren, E. et al. The macrophage migration inhibitory factor MIF is a phenylpyruvate tautomerase. FEBS letters 417 , 85-88 (1997).

66. Taylor, A. B. et al. Crystal structure of macrophage migration inhibitory factor complexed with (E)-2-fluoro-p-hydroxycinnamate at $1.8 \mathrm{~A}$ resolution: implications for enzymatic catalysis and inhibition. Biochemistry 38, 7444-7452, https://doi.org/10.1021/ bi9904048 (1999).

67. Ouertatani-Sakouhi, H. et al. Identification and characterization of novel classes of macrophage migration inhibitory factor (MIF) inhibitors with distinct mechanisms of action. The Journal of biological chemistry 285, 26581-26598, https://doi.org/10.1074/jbc. M110.113951 (2010).

68. Calandra, T. \& Roger, T. Macrophage migration inhibitory factor: a regulator of innate immunity. Nature reviews. Immunology 3 , 791-800, https://doi.org/10.1038/nri1200 (2003).

69. Mitchell, R. A. et al. Macrophage migration inhibitory factor (MIF) sustains macrophage proinflammatory function by inhibiting p53: regulatory role in the innate immune response. Proceedings of the National Academy of Sciences of the United States of America 99, 345-350, https://doi.org/10.1073/pnas.012511599 (2002).

70. Emonts, M. et al. Association between high levels of blood macrophage migration inhibitory factor, inappropriate adrenal response, and early death in patients with severe sepsis. Clinical infectious diseases: an official publication of the Infectious Diseases Society of America 44, 1321-1328, https://doi.org/10.1086/514344 (2007).

71. Shi, C. \& Pamer, E. G. Monocyte recruitment during infection and inflammation. Nature reviews. Immunology 11, 762-774, https:// doi.org/10.1038/nri3070 (2011).

72. Girard, E., Strathdee, C., Trueblood, E. \& Queva, C. Macrophage migration inhibitory factor produced by the tumour stroma but not by tumour cells regulates angiogenesis in the B16-F10 melanoma model. British journal of cancer 107, 1498-1505, https://doi. org/10.1038/bjc.2012.392 (2012).

73. Choudhary, S. et al. Macrophage migratory inhibitory factor promotes bladder cancer progression via increasing proliferation and angiogenesis. Carcinogenesis 34, 2891-2899, https://doi.org/10.1093/carcin/bgt239 (2013).

74. White, E. S. et al. Macrophage migration inhibitory factor and CXC chemokine expression in non-small cell lung cancer: role in angiogenesis and prognosis. Clinical cancer research: an official journal of the American Association for Cancer Research 9, 853-860 (2003).

75. Coleman, A. M. et al. Cooperative regulation of non-small cell lung carcinoma angiogenic potential by macrophage migration inhibitory factor and its homolog, D-dopachrome tautomerase. Journal of immunology (Baltimore, Md.: 1950) 181, 2330-2337 (2008).

76. Hudson, J. D. et al. A proinflammatory cytokine inhibits $\mathrm{p} 53$ tumor suppressor activity. The Journal of experimental medicine 190, 1375-1382, https://doi.org/10.1084/jem.190.10.1375 (1999).

77. Kleemann, R. et al. Intracellular action of the cytokine MIF to modulate AP-1 activity and the cell cycle through Jab1. Nature 408, 211-216, https://doi.org/10.1038/35041591 (2000).

\section{Acknowledgements}

We sincerely acknowledge the support provided by the Institute of Life Sciences, Bhubaneswar to SBS and D.V.; extramural funding by SERB, DST, India (EMR/2016/006214) to SBS; SERB National Post-Doctoral Fellowship to P.D.; and DBT Research Fellowship to R.S. Data collection at ESRF-ID30A-3 was facilitated by the DBT supported ESRF Access Program (BT/INF/22/SP22660/2017).

\section{Author contributions}

V.S., R.S. and P.D. contributed to the conception, design and execution of experiments; S.C.S. helped in the execution and analysis of tautomerase assay; D.M. and S.M. helped in manuscript writing and editing, and S.S. and D.V. contributed in conception, experimental design and supervision of the overall study.

\section{Competing interests}

The authors declare no competing interests.

\section{Additional information}

Supplementary information is available for this paper at https://doi.org/10.1038/s41598-019-51947-7.

Correspondence and requests for materials should be addressed to D.V. or S.S.

Reprints and permissions information is available at www.nature.com/reprints.

Publisher's note Springer Nature remains neutral with regard to jurisdictional claims in published maps and institutional affiliations. 
(c) (i) Open Access This article is licensed under a Creative Commons Attribution 4.0 International License, which permits use, sharing, adaptation, distribution and reproduction in any medium or format, as long as you give appropriate credit to the original author(s) and the source, provide a link to the Creative Commons license, and indicate if changes were made. The images or other third party material in this article are included in the article's Creative Commons license, unless indicated otherwise in a credit line to the material. If material is not included in the article's Creative Commons license and your intended use is not permitted by statutory regulation or exceeds the permitted use, you will need to obtain permission directly from the copyright holder. To view a copy of this license, visit http://creativecommons.org/licenses/by/4.0/.

(C) The Author(s) 2019 